\title{
Discovery of New Selective Butyrylcholinesterase (BChE) Inhibitors with Anti-A $\beta$ Aggregation Activity: Structure-Based Virtual Screening, Hit Optimization and Biological Evaluation
}

\author{
Cheng-Shi Jiang ${ }^{1, * \mathbb{D}}$, Yong-Xi Ge ${ }^{1}$, Zhi-Qiang Cheng ${ }^{1}$, Yin-Yin Wang ${ }^{1}$, Hong-Rui Tao ${ }^{1,2}$, \\ Kongkai Zhu ${ }^{1, *}$ and Hua Zhang ${ }^{1, *}$ (i) \\ 1 School of Biological Science and Technology, University of Jinan, Jinan 250022, China \\ 2 Drug Discovery and Design Center, State Key Laboratory of Drug Research, \\ Shanghai Institute of Meteria Medica, Chinese Academy of Sciences, Shanghai 201203, China \\ * Correspondence: bio_jiangcs@ujn.edu.cn (C.-S.J.); bio_zhukk@ujn.edu.cn (K.Z.); \\ bio_zhangh@ujn.edu.cn (H.Z.); Tel.: +86-0531-8973-6199 (H.Z.)
}

Received: 16 June 2019; Accepted: 12 July 2019; Published: 15 July 2019

check for updates

\begin{abstract}
In this study, a series of selective butyrylcholinesterase (BChE) inhibitors was designed and synthesized from the structural optimization of hit 1, a $4-((3,4$ -dihydroisoquinolin-2(1H)-yl)methyl)benzoic acid derivative identified by virtual screening our compound library. The in vitro enzyme assay results showed that compounds 9 ((4-((3,4-dihydroisoquinolin-2(1H)-yl)methyl)phenyl)(pyrrolidin-1-yl)methanone) and 23 ( $N$-(2 -bromophenyl)-4-((3,4-dihydroisoquinolin-2(1H)-yl)methyl)benzamide) displayed improved BChE inhibitory activity and good selectivity towards $\mathrm{BChE}$ versus $\mathrm{AChE}$. Their binding modes were probed by molecular docking and further validated by molecular dynamics simulation. Kinetic analysis together with molecular modeling studies suggested that these derivatives could target both the catalytic active site (CAS) and peripheral anionic site (PAS) of BChE. In addition, the selected compounds 9 and 23 displayed anti-A $\beta_{1-42}$ aggregation activity in a dose-dependent manner, and they did not show obvious cytotoxicity towards SH-SY5Y neuroblastoma cells. Also, both compounds showed significantly protective activity against $A \beta_{1-42}$-induced toxicity in a SH-SY5Y cell model. The present results provided a new valuable chemical template for the development of selective BChE inhibitors.
\end{abstract}

Keywords: selective BChE inhibitor; virtual screening; structural optimization; molecular dynamics; anti-A $\beta$ aggregation

\section{Introduction}

Alzheimer's disease (AD), one of the most common neurodegenerative disorders, accounts for about $60-80 \%$ of all cases of dementia [1,2]. According to the statistics, nearly 50 million people worldwide have $\mathrm{AD}$ or a related dementia, and the number of $\mathrm{AD}$ patients is expected to triple by 2050 [3]. AD has now been the third leading cause of death, outpaced only by cardiovascular diseases and cancer [4]. However, there is still no successful therapy or drug to reverse or even slow the course of this disease [5].

Although the pathogenesis of AD is complex and not fully understood, several important clinical hallmarks, such as low level of acetylcholine (ACh), beta-amyloid (A $\beta$ ) protein aggregation, and tau $(\tau)$-protein phosphorylation, are involved in the occurrence and development of $\mathrm{AD}$ [6]. In recent years, therapies for anti-AD primarily focused on $\mathrm{A} \beta$ and tau have received more attention [7], however, 
various $A \beta$ - and tau-targeting agents have failed in clinical trials [8]. Based on cholinergic dysfunction hypothesis, increasing the level of ACh in the brain to improve cholinergic neurotransmission is still the most effective therapy for AD treatment [9]. The ACh in the brain can be mainly hydrolyzed two types of cholinesterases (ChEs), namely acetylcholinesterase (AChE) and butyrylcholinesterase (BChE) [10]. In the brain of healthy adults, AChE is responsible for $80 \%$ of the ACh activity, nearly $10^{13}$ fold more active than BChE [11]. Four AChE inhibitors (tacrine, donepezil, galantamine, and rivastigmine) have been clinically employed for the management of AD [12]. These drugs have beneficial effects on improving the cognitive and memorial functions of AD patients at mild and moderate stages, but tacrine was withdrawn from the market in 2013 due to its hepatotoxicity and the others drugs cannot stop or reverse the course of AD. In addition, the side effects (e.g., nausea, vomiting, diarrhea) of AChE inhibitors also limit the administered doses of these drugs [13].

Although BChE played a minor role in regulating the brain ACh level in the health brain, increasing experimental evidence demonstrated that the function of $\mathrm{BChE}$ in termination of cholinergic neurotransmission significantly changed in brains of patients with progressive AD. In advanced AD patients, the activity of BChE progressively increases while the AChE activity remains unchanged or declines [14-16]. Moreover, BChE was believed to compensate the function of AChE based on the experiment that $\mathrm{AChE}$ knockout mice could still survive with the normal level and localization of BChE [17,18], while silent BChE mutations in humans showed a slower rate of cognitive decline [19]. Therefore, inhibition of BChE was also expected to contribute to ameliorating a cholinergic effect. This hypothesis was in agreement with the experiment that selective inhibition of BChE could significantly raise ACh levels and improve the cognitive performance of aged rats, without any adverse parasympathetic side effects, which are known to be the defect of traditional AChE inhibitors [15,20]. Taken together, selective inhibition on BChE could represent a promising clinical treatment strategy for AD.

However, the development of inhibitors selectively targeting BChE over AChE is a challenging task, since the two isoforms hAChE and hBChE are highly homologous proteins [21]. Although many structurally diverse selective BChE inhibitors, such as carbamate-based compounds [22], paltanic acid derivatives [23], naphthamide compounds [24], phenserine-based compounds [25], and isosorbide analogues [26], have been reported, very few of them (bisnorcymserine and eptastigmine) have entered into clinical trials $[16,27]$. Thus, the discovery of effective selective BChE inhibitors with novel molecular skeletons is urgently needed. Virtual screening has been proven to be a very powerful approach for discovering new drug hits with desired properties and structural diversity [28], while a structure-activity relationship (SAR) study based on enzyme or cell bioassay has emerged as the most versatile to in provide useful information for structural modification and improvement $[29,30]$.

In our project for discovering new ChE inhibitors [31-36], a new selective BChE inhibitor 1 $\left(\mathrm{BChE}, \mathrm{IC}_{50}=17.94 \pm 1.86 \mu \mathrm{M} ; \mathrm{AChE}, \mathrm{IC}_{50}>50 \mu \mathrm{M}\right)$ (Figure 1 and Table 1$)$ was recently identified from our in-house compound library by performing virtual screening and in vitro BChE/AChE assay. The follow-up molecular docking-guided chemical optimization and SAR study starting from hit 1 resulted in some new potent selective BChE inhibitors. Among them, compounds 9 and 23 showed improved BChE inhibitory activity by 9 and 21 folds compared with 1. In addition, both compounds 9 and 23 displayed anti-A $\beta$ aggregation activity and nontoxicity against SH-SY5Y cells. Herein, we describe a series of new selective BChE inhibitors with anti-A $\beta$ aggregation activity using the combination of virtual screening/molecular, synthesis and in vitro biological evaluation, as well as the molecular dynamics simulation study on the most potent compound 23.

\section{Results and Discussion}

\subsection{Molecular Docking-Based Virtual Screening to Identify Hit 1 as Promising Selective BChE Inhibitor}

The BChE:tacrine complex crystal structure (PDB entry: 4BDS) [37] was used to perform molecular docking-based virtual screening. Residues in BChE centered on tacrine (15 $\AA$ ) were defined as a binding 
pocket. An in-house database, containing 1225 compounds with different scaffold was screened in silico to identify the active compounds against $\mathrm{BChE}$. The docking score of tacrine redocked to $\mathrm{BChE}$ was -8.498 , while the docking scores of the top- 100 compounds was higher than that of tacrine, so the top-100 compounds ranked according to the docking score were obtained for the following visual selection. After clustering the 100 compounds with "Clustering Molecules" protocols inserted in Pipeline Plot 7.5, we finally selected 30 compounds for experimental validation. Among them, compound 1 , showed highest inhibitory activity against $\mathrm{BChE}$ with $\mathrm{IC}_{50}$ value of $17.94 \pm 1.86 \mu \mathrm{M}$, without obvious activity towards $\mathrm{AChE}$ (inhibitory ratio $=26.6 \%$ at $50 \mu \mathrm{M}$ ). A literature survey showed that compound $\mathbf{1}$ was a new compound that was structurally different from the previously reported BChE inhibitor, which highlighted that compound $\mathbf{1}$ was used as a hit compound for subsequent structural optimization.
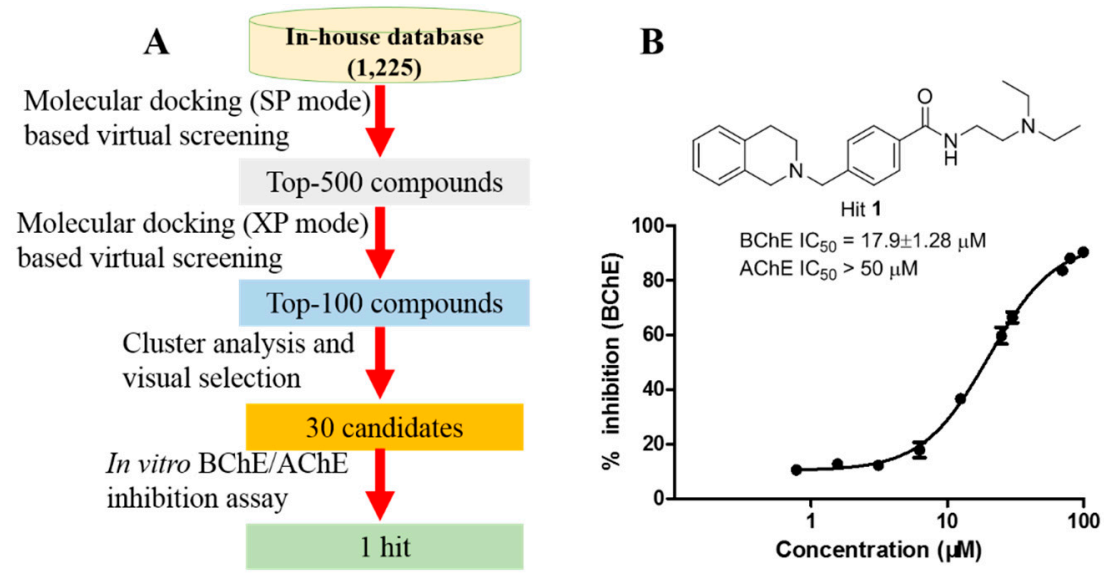

Figure 1. In silico screening procedure and ChE assay results of hit 1. (A) The discovery workflow of the hit compound. (B) Chemical structure of hit $\mathbf{1}$ and its ChE inhibitory activity.

\subsection{Binding mode of 1 Provides Insights for Hit Optimization}

In order to get guidance for rational structural optimization of hit 1 , the proposed binding mode of 1 with the active sites of $\mathrm{BChE}$ was explored using a molecular docking method. As depicted in Figure 2A, compound 1 fitted well in the active-site gorge of $\mathrm{BChE}$, and its tertrahydroisoquinoline fragment was located in the catalytic active site (CAS) which had spatial overlapping with tacrine, while the amino side-chain was oriented towards the peripheral anionic site (PAS) of BChE. Figure 2B showed the important interactions involved between compound $\mathbf{1}$ and the residues of $\mathrm{BChE}$, including $\pi-\pi$ and cation- $\pi$ interactions of tertrahydroisoquinoline fragment with W82 (a key residue in the CAS) and $\pi-\pi$ interaction of benzene fragment with Y332 (a key residue in the PAS of BChE). Since the interactions with W82 and Y332 play an important role in BChE inhibition, the two key fragments (tertrahydroisoquinoline and benzene) should be retained before performing chemical modifications. With this in mind, the amino side-chain was then rationally modified based on the above molecular docking result in order to improve its inhibitory activity against BChE. 


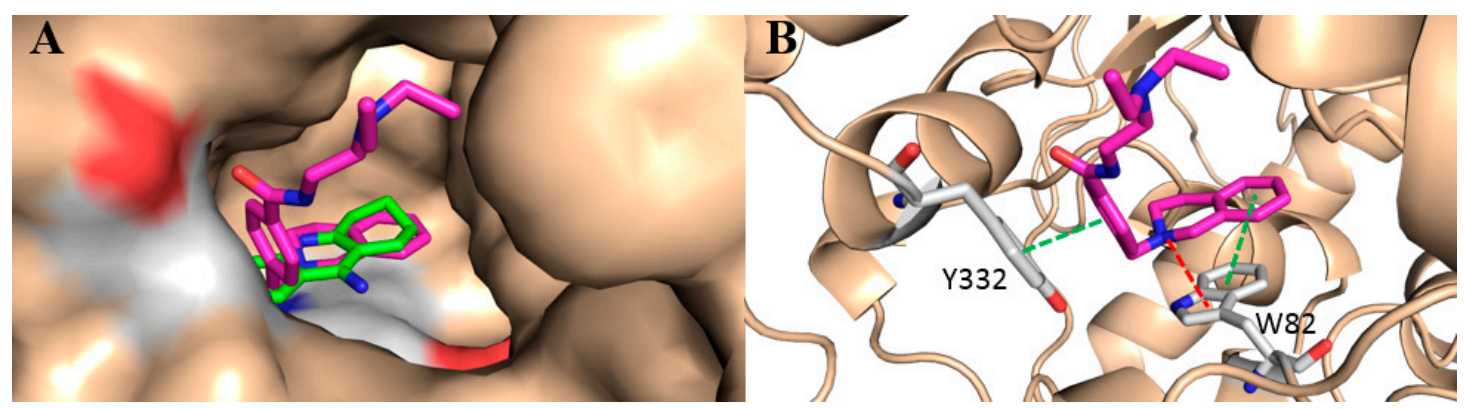

Figure 2. Proposed binding mode of hit $\mathbf{1}$ with BChE. (A) The predicted orientation of $\mathbf{1}$ (carbon in magenta) in the active pocket of BChE (PDB: 4BDS) with tacrine as ligand in green color; (B) The key interactions of 1 with residues in the active sites of BChE. $\pi-\pi$ stacking and cation- $\pi$ interaction are displayed in green and red dashed lines, respectively.

\subsection{Chemcial Synthesis}

To improve the potency of hit 1 and to discuss the structure-activity relationship (SAR), a range of amide and ester derivatives of 4-((3,4-dihydroisoquinolin-2(1H)-yl)methyl)benzoic acid were designed and synthesized based on molecular docking analysis. The synthetic route outlined in Scheme 1 started from the commercial tertrahydroisoquinoline or its derivatives $\mathrm{A}$, which reacted with 4-(bromomethyl)benzoic acid to give intermediates B. Subsequent condensation reaction of acids $B$ with various alkyl amines, aromatic amines, phenol, thiophenol or tryptamines smoothly yielded the target compounds $\mathbf{1 - 3 0}$.

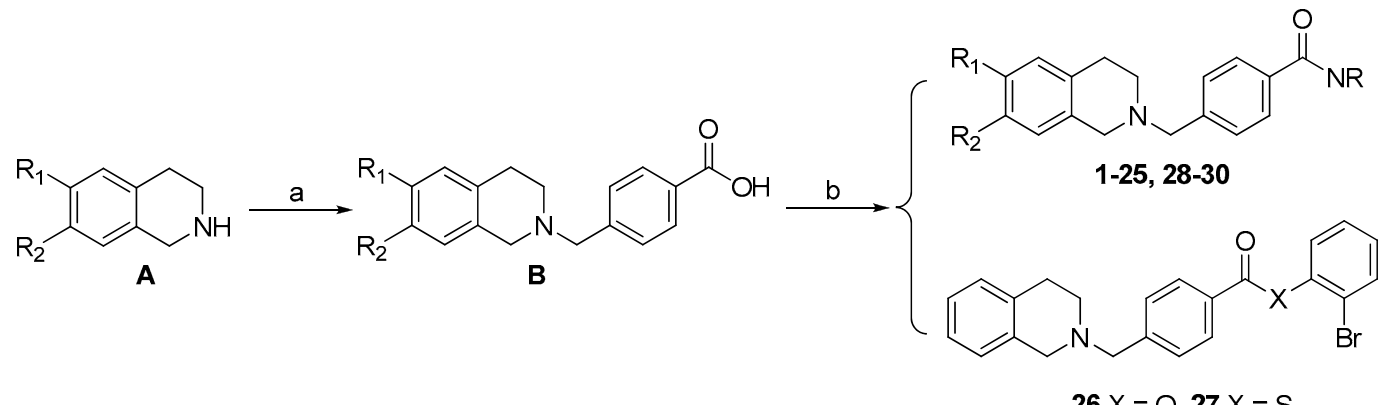

Scheme 1. Synthesis of compounds 1-30. (a) 4-(bromomethyl)benzoic acid or its derivatives, THF, reflux, $8 \mathrm{~h}$; (b) agents including alkyl amines, aromatic amines, phenol, thiophenol or tryptamines, HATU, DIPEA, DMF, r.t., 8 h.

\subsection{Biological Evaluation and SAR Study}

The Ellman's method was used to determine all the compounds' BChE and AChE inhibitory activities, and tacrine was used as the reference control. The SAR investigation was mainly focused on the amino side-chain in the first round of structural modification. From Table 1, modification of the terminal amino group in compound $\mathbf{1}$ using homologue (compounds 2-4) or isostere (compound 5) led to a significant loss of BChE inhibitory activity or selectivity towards BChE. Scaffold hopping-based cyclization of the diethylamino group was tried to yield analogs 6-10, of which the compounds 7-9 possessing tetrahydropyrrole ring showed improved inhibition and selectivity towards $\mathrm{BChE}$, with the best $\mathrm{IC}_{50}$ value of $2.68 \pm 0.28 \mu \mathrm{M}$ for 9 . 
Table 1. The inhibitory activities of compounds $1-10$ against ChEs.

\begin{tabular}{|c|c|c|c|c|c|}
\hline \multirow{2}{*}{ No. } & \multirow{2}{*}{$\mathbf{R}$} & \multicolumn{2}{|c|}{ BChE } & \multicolumn{2}{|r|}{ AChE } \\
\hline & & $I^{a}(\%)$ & $\mathrm{IC}_{50}(\mu \mathrm{M})$ & $\operatorname{IR}(\%)$ & $\mathrm{IC}_{50}(\mu \mathrm{M})$ \\
\hline 1 & & 68.4 & $17.9 \pm 1.86$ & 26.6 & $-\mathrm{b}$ \\
\hline 2 & & 76.4 & $9.98 \pm 0.89$ & 56.1 & $21.46 \pm 0.98$ \\
\hline 3 & & 36.9 & - & 11.4 & - \\
\hline 4 & & 27.2 & - & 8.8 & - \\
\hline 5 & & 56.6 & $38.10 \pm 1.68$ & 2.7 & - \\
\hline 6 & & 19.1 & - & 13.4 & - \\
\hline 7 & & 45.4 & $15.2 \pm 2.33$ & 4.4 & - \\
\hline 8 & & 63.0 & $24.98 \pm 1.27$ & 13.3 & - \\
\hline 9 & & 95.1 & $2.68 \pm 0.28$ & 29.1 & - \\
\hline 10 & & 11.2 & - & 3.4 & - \\
\hline Tacrine & & 98.4 & $0.14 \pm 0.01$ & 96.2 & $0.11 \pm 0.03$ \\
\hline
\end{tabular}

$\mathrm{IC}_{50}$ values are expressed as mean $\pm \mathrm{SD}$ of three separate experiments. ${ }^{\text {a }}$ Inhibition ratio (IR) against $\mathrm{ChE}$ at $50 \mu \mathrm{M}$. b Not tested.

With compound 9 in hand, the substituent effect at C-6 and C-7 of tetrahydroisoquinoline on the BChE inhibitory activity was studied through the evaluation of analogs $11-14$. The results in Table 2 indicated that both monosubstitution and disubstitution made the BChE inhibitory activity and selectivity decrease, compared with $\mathbf{9}$, suggesting that it might not accommodate the substitution at C-6 and C-7 of a tetrahydroisoquinoline fragment.

Table 2. The inhibitory activities of compounds 11-14 against ChEs.

\begin{tabular}{|c|c|c|c|}
\hline No. & & $\mathrm{BChE} \mathrm{IC}_{50}(\mu \mathrm{M})$ & $\mathrm{AChE} \mathrm{IC}_{50}(\mu \mathrm{M})$ \\
\hline 11 & $\mathrm{R}_{1}=\mathrm{Br}, \mathrm{R}_{2}=\mathrm{H}$ & $8.38 \pm 0.65$ & $30.61 \pm 1.82$ \\
\hline 12 & $\mathrm{R}_{1}=\mathrm{H}, \mathrm{R}_{2}=\mathrm{Br}$ & $39.44 \pm 2.25$ & $>50$ \\
\hline 13 & $\mathrm{R}_{1}=\mathrm{OMe}, \mathrm{R}_{2}=\mathrm{H}$ & $12.62 \pm 0.88$ & $12.13 \pm 0.90$ \\
\hline 14 & $\mathrm{R}_{1}=\mathrm{OMe}, \mathrm{R}_{2}=\mathrm{OMe}$ & - & $18.24 \pm 0.86$ \\
\hline
\end{tabular}

Next, the tetrahydroisoquinoline ring was unchanged and the tetrahydropyrrole group was later replaced by various benzene groups. The results were shown in Table 3 . Among compounds 15-18, only one compound (16) with 2-fluoroanilino group showed $\mathrm{BChE}$ activity $\left(\mathrm{IC}_{50}=6.9 \mu \mathrm{M}\right)$, indicating that 
2-subsititution at benzene ring might be optimal for BChE activity and 3-/4-subsititution was inadvisable. Notably, replacement of 2-F by 2-Br led to an obvious increase of the activity of 23 by about 3- and 8-fold compared with that of 9 and 16, respectively. Changing the position of $\mathrm{Br}$ from C-2 to C-3 (24) or C-4 (25) obviously made its BChE inhibitory activity lose, which was in accordance with the SAR obtained from compounds 16-18. Then, replacement of 2-bromoanilino moitey in compound 23 by 2-bromophenol (26) or 2-bromophenylthio (27) also led to a significant loss of activity, suggesting that the amide group takes an important role in its activity. Recently, Mahdavi et al. reported a group of potent selective tryptamine-derived BChE inhibitors [38], thus the effect of tryptamine fragment fused into our compounds 28-30 on BChE activity was evaluated. Unfortunately, all these three target compounds were inactive toward BChE.

Table 3. The inhibitory activities of compounds $\mathbf{1 5 - 3 0}$ against ChEs.

\begin{tabular}{|c|c|c|c|c|c|}
\hline \multirow{2}{*}{ No. } & \multirow{2}{*}{$\mathbf{R}$} & \multicolumn{2}{|c|}{ BChE } & \multicolumn{2}{|c|}{ AChE } \\
\hline & & $\operatorname{IR}^{\mathrm{a}}(\%)$ & $\mathrm{IC}_{50}(\mu \mathrm{M})$ & $\operatorname{IR}(\%)$ & $\mathrm{IC}_{50}(\mu \mathrm{M})$ \\
\hline 15 & & 23.7 & $-\mathrm{b}$ & 8 & - \\
\hline 16 & & 74.7 & $6.93 \pm 0.48$ & 2.1 & - \\
\hline 17 & & 10.0 & - & 7.7 & - \\
\hline 18 & & 14.1 & - & 7.0 & - \\
\hline 19 & & 83.9 & $14.78 \pm 0.58$ & 2.3 & - \\
\hline 20 & & 13.2 & - & 21.8 & - \\
\hline 21 & & 38.6 & - & 5 & - \\
\hline 22 & & 15.4 & - & 28.3 & - \\
\hline 23 & & 97.9 & $0.83 \pm 0.08$ & 22.7 & - \\
\hline 24 & & 35.1 & - & 21.6 & - \\
\hline 25 & & 8.1 & - & 40.6 & - \\
\hline
\end{tabular}


Table 3. Cont.

\begin{tabular}{|c|c|c|c|c|c|}
\hline \multirow{2}{*}{ No. } & \multirow{2}{*}{$\mathbf{R}$} & \multicolumn{2}{|c|}{ BChE } & \multicolumn{2}{|c|}{ AChE } \\
\hline & & $I^{a}{ }^{a}(\%)$ & $\mathrm{IC}_{50}(\mu \mathrm{M})$ & IR(\%) & $\mathrm{IC}_{50}(\mu \mathrm{M})$ \\
\hline 26 & & 11 & - & 26.2 & - \\
\hline 27 & & 1.5 & - & 22.8 & - \\
\hline 28 & & 1.3 & - & 8.4 & - \\
\hline 29 & & 6.5 & - & 4.6 & - \\
\hline 30 & & 1.1 & - & 8.3 & - \\
\hline
\end{tabular}

$\mathrm{IC}_{50}$ values are expressed as mean $\pm \mathrm{SD}$ of three separate experiments. ${ }^{a}$ Inhibition ratio (IR) against ChE at $50 \mu \mathrm{M}$.

b Not tested.

\subsection{Kinetic Study on BChE Inhibition}

To further understand the action mechanism for BChE inhibition of this series of compounds, the kinetic assay was performed with the optimal compound $\mathbf{2 3}$ as a model compound. The enzyme kinetic result reveals that increasing slope (decreasing $V_{\max }$ ) and increased $K_{\max }$ with increasing concentrations of $\mathbf{2 3}$ (Figure 3A). This pattern clearly indicates compound 23 is mixed-type inhibitor that simultaneously interacts with both CAS and PAS of BChE. The result was confirmed by the subsequent molecular docking study of $\mathbf{2 3}$. Replots of the slope against the concentrations of $\mathbf{2 3}$ indicates that the $K_{\mathrm{I}}$ value is $0.52 \mu \mathrm{M}$, which fits its $\mathrm{IC}_{50}$ value for $\mathrm{BChE}$ inhibition.
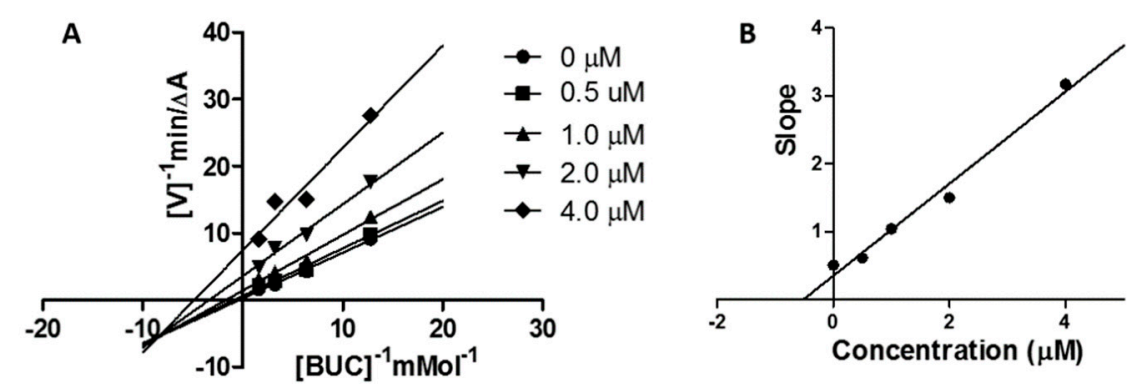

Figure 3. Kinetic study of 23 on BChE inhibition. (A) The Lineweaver-Burk reciprocal plots records enzyme velocity curves-substrate concentration in the presence of $\mathbf{2 3}$ at different concentrations;

(B) Plot of slopes versus the concentrations of 23.

\subsection{Binding Mode Analysis}

A probable binding mode of $\mathbf{2 3}$ with $\mathrm{BChE}$ was obtained by conducting molecular docking. As illustrated in Figure 4A, compound 23 could well occupy the tacrine binding site and displayed similar binding mode with tacrine. In addition, the bromophenyl of 23 extended outside of tacrine binding site. As we can see from Figure $4 \mathrm{~B}, \mathrm{C}$, the binding pocket of $\mathbf{2 3}$ was a hydrophobic pocket 
composed of D70, W82, G115, G116, Y128, E197, P285, A328, F329, Y332, and H438 residues, and the $\pi-\pi$ interactions of 23 with W82 and Y332 were retained. Hydrophobic interaction is the main interaction in stabilizing 23 to the tacrine binding site.

$\mathbf{A}$

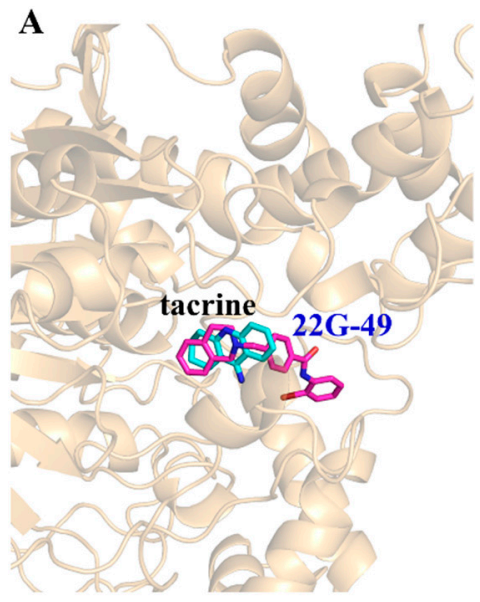

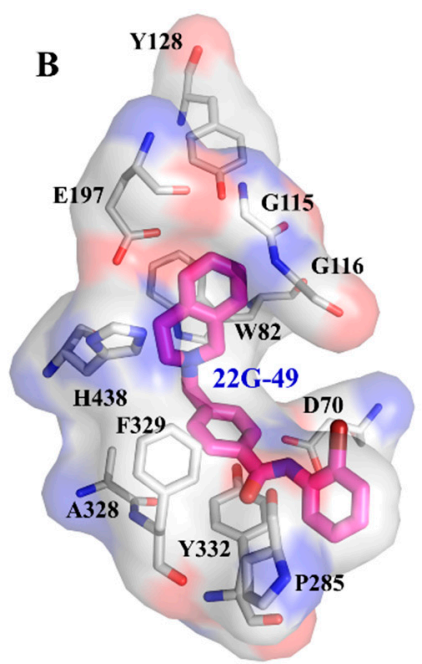

Figure 4. Predicted binding mode of 23 with BChE. (A) Superimposition of the binding modes of 23 and tacrine. $\mathrm{BChE}, 23$ and tacrine are shown as light-orange cartoon, magenta, and cyan sticks. (B) Detailed interactions between 23 and BChE. Compound 23 is shown as magenta sticks, while the carbon atoms of the interacting residues are colored in white. (C) Key interactions involved in stabilizing 23 at the tacrine binding site.

\subsection{Molecular Dynamics}

To further validate the binding mode of 23 with BChE, $100 \mathrm{~ns}$ molecular dynamics (MD) simulation was employed on the complex model of BChE: 23 that was constructed using molecular docking. The root-mean-square deviations (RMSDs) values for the heavy atoms of BChE and 23 (Figure S1) were steady, which indicated that MD trajectory is reliable for further analysis. Then the binding free energy of $\mathbf{2 3}$ with BChE was calculated by Molecular Mechanics Poisson-Boltzmann Surface Area (MM-PBSA) method, as shown in Table 4, the value $(-25.93 \mathrm{kcal} / \mathrm{mol})$ indicated a stable binding of 23 with BChE. Besides, based on MD trajectories, the occupancy rate of hydrophobic interactions between 23 and the active site residues was calculated and shown in Table 5. This result indicated that all the hydrophobic interactions between 23 and BChE could be well retained during MD simulation.

Table 4. The binding free energy calculated by MM-PBSA method. $\Delta \mathrm{G}_{\text {gas }}$ represents the binding free energy in vacuum and $\Delta \mathrm{G}_{\text {solv }}$ represents the solvation free energy changes. $\Delta \mathrm{G}=\Delta \mathrm{G}_{\mathrm{gas}}+\Delta \mathrm{G}_{\text {solv }}$. The data were shown in $\mathrm{kcal} / \mathrm{mol}$.

\begin{tabular}{cccc}
\hline Inhibitor & $\Delta \mathbf{G}_{\text {gas }}$ & $\Delta \mathbf{G}_{\text {solv }}$ & $\Delta \mathbf{G}$ \\
\hline $\mathbf{2 3}$ & $-59.52 \pm 10.41$ & $33.59 \pm 9.06$ & $-25.93 \pm 5.07$ \\
\hline
\end{tabular}


Table 5. The hydrophobic interactions residues in BChE and their corresponding occupancy rates.

\begin{tabular}{cccc}
\hline Residue & Occupancy Rate (\%) & Residue & Occupancy Rate (\%) \\
\hline D70 & 79 & P285 & 61 \\
\hline W82 & 100 & A328 & 57 \\
\hline G115 & 58 & F329 & 80 \\
\hline G116 & 56 & Y332 & 78 \\
\hline Y128 & 68 & H438 & 59 \\
\hline E197 & 55 & & \\
\hline
\end{tabular}

\subsection{Inhibition on $A \beta$ Aggregation}

The $A \beta$ peptide aggregation in the brain is known to trigger the onset of neurodegenerative disease and considered as a key pathological hallmark of $\operatorname{AD}[39,40]$. The aggregation and accumulation of the $\mathrm{A} \beta$ peptide could form oligomers and plaques, which is a step-by-step process starting from a soluble monomer and proceeding through oligomeric structures, protofibrils, and eventually amyloid fibrils. Of them, the soluble oligomers are the more toxic species [41,42]. BChE was found to be enriched within $\mathrm{A} \beta$ plaques in brain of $\mathrm{AD}$ patients and to promote the formation of $\mathrm{A} \beta$ fibrils, while $\mathrm{BChE}$-knockout could reduce $A \beta$ deposition in brain of AD mouse model $[43,44]$. Thus, $B C h E$ inhibitor could reduce the $A \beta$ aggregation. Based on these observations, inhibitors 9 and 23 were evaluated for their in vitro ability to inhibit $A \beta$ aggregation using the thioflavin $\mathrm{T}(\mathrm{ThT}$ ) method. Donepezil was used as a positive control, and the results was shown in Figure 5. Both compounds exhibited anti-A $\beta_{1-42}$ aggregation activity in a dose-dependent manner from the concentration of 1 to $10 \mu \mathrm{M}$. Especially, the inhibition ratios of 9 and 23 (at $10 \mu \mathrm{M}$ ) towards $A \beta_{1-42}$ aggregation are $45.5 \pm 6.2 \%$ and $44.2 \pm 3.3 \%$, respectively, indicating that the activity of both compounds are significantly more potent than that of donepezil $(25.8 \pm 6.2 \%$ at $10 \mu \mathrm{M})$.

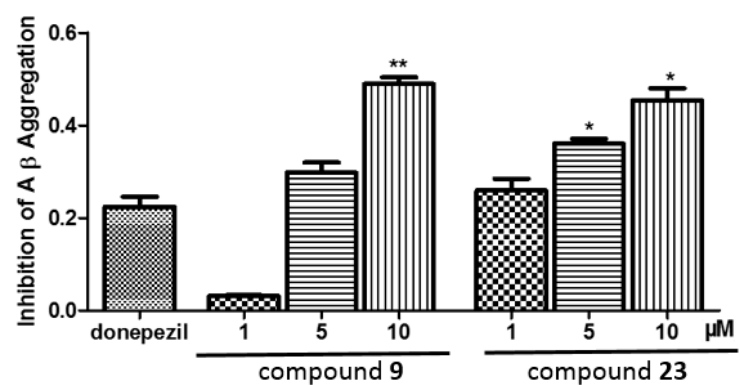

Figure 5. Inhibitory activity against $A \beta_{1-42}$ produced by $9(1,5,10 \mu \mathrm{M}), \mathbf{2 3}(1,5,10 \mu \mathrm{M})$, and donepezil $(10 \mu \mathrm{M})$. Data are shown as mean $\pm \operatorname{SD}(n=3)$, with $* p<0.05$ and ${ }^{* *} p<0.01$ versus donepezil.

\subsection{Cell Viability Assay}

Next, to determine the potential cytotoxic activity of selected compounds $\mathbf{9}$ and $\mathbf{2 3}$ towards SH-SY5Y neuroblastoma cells, the viability of cells treated by 9 and 23 was evaluated by MTT assay. The result presented in Figure 6 revealed that both compounds did not show obvious cytotoxicity in the range of tested concentration, even at $200 \mu \mathrm{M}$. 


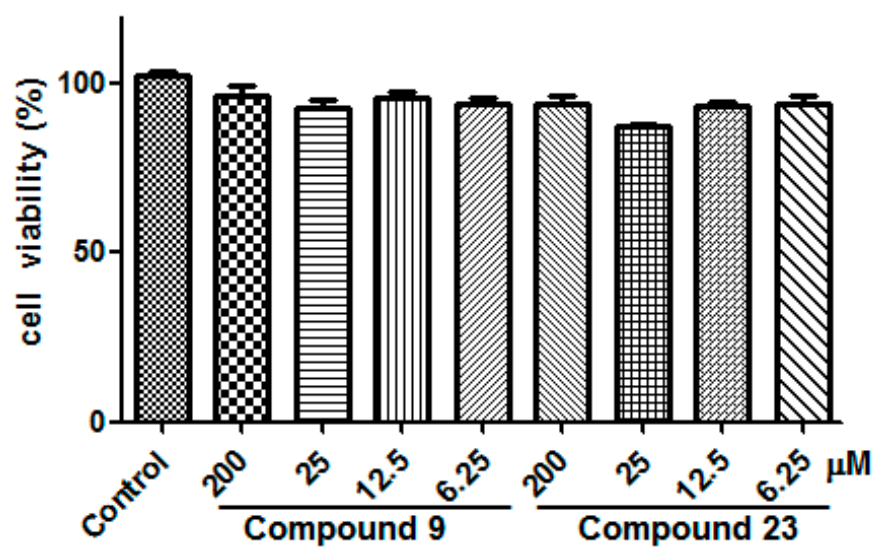

Figure 6. The cytotoxicity of $\mathbf{9}$ and $\mathbf{2 3}$ against SH-SY5Y cells. Date are expressed as the percent variation with respect to the viability recorded in control cells.

\subsection{Protective Activity of 9 and 23 Against A $\beta_{1-42}$-Induced Toxicity in SH-SY5Y Cells}

Since compounds 9 and 23 showed anti-A $\beta_{1-42}$ aggregation activity and nontoxicity towards SH-SY5Y cells, their in vitro ability to protect SH-SY5Y cells from $A \beta_{1-42}$-induced toxicity was evaluated using the MTT method with epigallocatechin gallate (EGCG) as a positive control. As shown in Figure 7, after the SH-SY5Y cells was exposed to $10 \mu \mathrm{M} \mathrm{A} \beta_{1-42}$ for $24 \mathrm{~h}$, the cell viability of $\mathrm{A} \beta_{1-42}$-treated group decreased to $63.21 \pm 1.30 \%$, while in compound-treated groups both 9 and 23 could significantly protect $\mathrm{SH}-\mathrm{SY} 5 \mathrm{Y}$ cells from $\mathrm{A} \beta_{1-42}$-induced toxicity compared with the $\mathrm{A} \beta$-injured group, by increasing cell viability to $84.74 \pm 1.77 \%$ and $91.14 \pm 1.25$ at $5 \mu \mathrm{M}$, and $88.80 \pm 0.81 \%$ and $98.04 \pm 1.70$ at $10 \mu \mathrm{M}$, respectively. More importantly, the protective activity of compound 23 was found to be significantly better than that of the positive control EGCG $(87.18 \pm 1.29 \%)$ at $10 \mu \mathrm{M}$. Based on this observation, compound 23 might be a more promising protective agent against $\mathrm{A} \beta_{1-42}$-induced toxicity on the SH-SY5Y cell model.

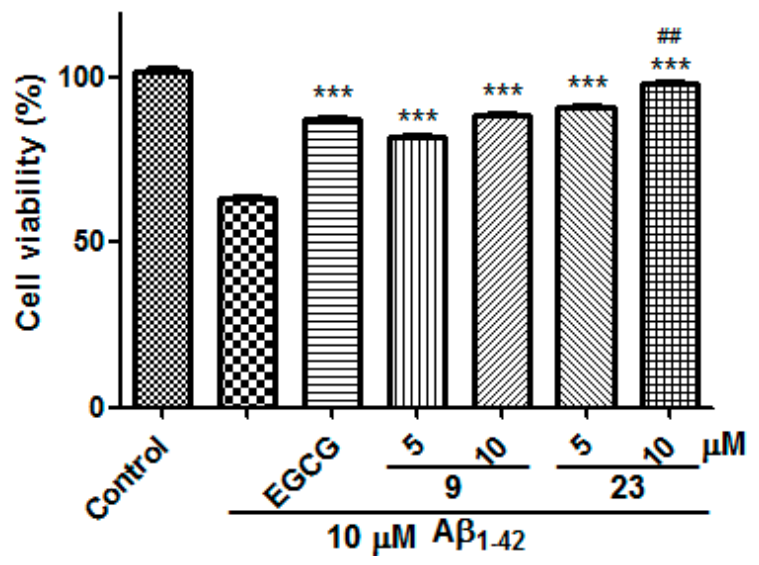

Figure 7. Protective effects of compounds 9 and 23 on $A \beta_{1-42}$-induced toxicity in SH-SY5Y cells. Data are expressed as mean $\pm \operatorname{SD}(n=3),{ }^{* * *} p<0.001$ versus $\mathrm{A} \beta_{1-42}$ treated only, and ${ }^{\# \#} p<0.01$ versus positive control EGCG.

\section{Materials and Methods}

\subsection{Virtural Screening}

An in-house database containing 1225 compounds was screened in silico against the BChE protein structure (PDB code: 4BDS) [37] through the GLIDE 5.5 software $[45,46]$. The BChE protein coordinates were first processed with the Protein Preparation Wizard Workflow inserted in Maestro, and the default settings were used for this step. Then the docking grids were created by defining 
residues within $15 \AA$ centered on tacrine in BChE. The parameters for the cutoff, neutralization, scaling, etc. were adopted with default settings. All the in-house database compounds were docked into the defined binding pocket and were ranked by G-score. The top 500 compounds were selected for further molecular docking validation with the extra precision (XP) mode to further enrich the potential active compounds [47]. Finally, after clustering, visual selection was made to yield the 30 candidate compounds, which were subjected to the BChE inhibition assay.

\subsection{Chemistry}

\subsubsection{General Methods}

Commercially available reagents were used without further purification. Organic solvents were evaporated with reduced pressure using a Büchi R-100 evaporator (Büchi, Flawil, Switzerland). Reactions were monitored by TLC using Yantai Jingyou (Yantai, China) GF254 silica gel plates. Silica gel column chromatography was performed on Biotage Isolera One (Biotage, Uppsala, Sweden) using silica gel (200-300 mesh) from Qingdao Hailang (Qingdao, China). NMR spectra were measured on Bruker Avance III $600 \mathrm{MHz}$ spectrometers (Bruker, Fällanden, Switzerland). Chemical shifts were expressed in $\delta(\mathrm{ppm})$ and coupling constants $(J)$ in $\mathrm{Hz}$ with solvent signals as internal standards $\left(\mathrm{CDCl}_{3}, \delta_{\mathrm{H}} 7.26 \mathrm{ppm}\right.$ and $\delta_{\mathrm{C}} 77.0 \mathrm{ppm}$; DMSO- $\delta_{6}, \delta_{\mathrm{H}} 2.50 \mathrm{ppm}$ and $\left.\delta_{\mathrm{C}} 39.5 \mathrm{ppm}\right)$. ESI-MS (electrospray ionization mass spectrometry) analyses were performed on an Agilent 1260-6460 Triple Quard LC-MS instrument (Agilent, Waldbronn, Germany), and HR-ESIMS data were acquired on an Agilent Q-TOF 6520 (Agilent, Waldbronn, Germany).

\subsubsection{General Procedure for the Synthesis of B}

The mixtures of 4-(bromomethyl)benzoic acid ( $200 \mathrm{mg}, 0.93 \mathrm{mmol}, 1$ equiv.) and corresponding tetrahydroisoquinolines A (1.86 mmol, 2 equiv.) in $8 \mathrm{~mL}$ anhydrous THF were refluxed for $8 \mathrm{~h}$, and the progress of the reaction was followed using TLC. After completion of the reaction, the reaction mixture was cooled to room temperature and the solvent was removed under vacuo. The resultant solid was dissolved in $1 \mathrm{~N} \mathrm{NaOH}(20 \mathrm{~mL})$ and then extracted with $\mathrm{CH}_{2} \mathrm{Cl}_{2}(3 \times 20 \mathrm{~mL})$. The aqueous layer was acidified to $\mathrm{pH}=1$ using $10 \% \mathrm{HCl}(20 \mathrm{~mL})$, resulting in precipitation of the final compounds.

4-((3,4-dihydroisoquinolin-2(1H)-yl)methyl)benzoic acid (B-1). Yellow solid (yield 90\%). ${ }^{1} \mathrm{H}-\mathrm{NMR}(600 \mathrm{MHz}$, DMSO- $\left.d_{6}\right) \delta 11.54$ (brs, $\left.1 \mathrm{H}, \mathrm{COOH}\right), 8.02(\mathrm{~d}, J=8.2 \mathrm{~Hz}, 2 \mathrm{H}), 7.82(\mathrm{~d}, J=8.2 \mathrm{~Hz}, 2 \mathrm{H}), 7.28-7.15(\mathrm{~m}, 4 \mathrm{H})$, $4.53(\mathrm{~d}, J=8.5 \mathrm{~Hz}, 2 \mathrm{H}), 4.29(\mathrm{~d}, J=18.0 \mathrm{~Hz}, 2 \mathrm{H}), 3.63(\mathrm{~s}, 2 \mathrm{H}), 3.03(\mathrm{~s}, 2 \mathrm{H}) .{ }^{13} \mathrm{C}-\mathrm{NMR}\left(150 \mathrm{MHz}, \mathrm{DMSO}-d_{6}\right)$ $\delta 166.9,134.5,131.8,131.7,131.5,129.7,128.6,128.3,127.7,126.7,126.6,57.8,51.5,48.6,39.5,24.9$. ESI-MS $m / z 268.1[\mathrm{M}+\mathrm{H}]^{+}$.

4-((6-bromo-3,4-dihydroisoquinolin-2(1H)-yl)methyl)benzoic acid (B-2). Yellow solid (yield 92.3\%). ${ }^{1} \mathrm{H}-\mathrm{NMR}$ $\left(600 \mathrm{MHz}, \mathrm{DMSO}-d_{6}\right) \delta 11.54(\mathrm{brs}, 1 \mathrm{H}, \mathrm{COOH}), 8.02(\mathrm{~d}, J=7.9 \mathrm{~Hz}, 2 \mathrm{H}), 7.83-7.77(\mathrm{~m}, 2 \mathrm{H}), 7.49(\mathrm{~s}, 1 \mathrm{H})$, $7.42(\mathrm{~d}, J=8.3 \mathrm{~Hz}, 1 \mathrm{H}), 7.16(\mathrm{~d}, J=8.3 \mathrm{~Hz}, 1 \mathrm{H}), 4.52(\mathrm{~d}, J=18.9 \mathrm{~Hz}, 2 \mathrm{H}), 4.26(\mathrm{~s}, 2 \mathrm{H}), 3.33-3.20(\mathrm{~m}, 3 \mathrm{H})$, $3.06(\mathrm{~s}, 1 \mathrm{H}) .{ }^{13} \mathrm{C}-\mathrm{NMR}\left(150 \mathrm{MHz}, \mathrm{DMSO}-d_{6}\right) \delta 166.9,134.3,131.8,131.6,131.1,129.7,129.5,128.9,127.8$, 120.6, 57.7, 51.0, 48.1, 39.5, 24.6. ESI-MS $m / z 346.0[\mathrm{M}+\mathrm{H}]^{+}$.

4-((7-bromo-3,4-dihydroisoquinolin-2(1H)-yl)methyl)benzoic acid (B-3). White solid (yield 87.5\%). ${ }^{1} \mathrm{H}-\mathrm{NMR}$ $\left(600 \mathrm{MHz}, \mathrm{DMSO}-d_{6}\right) \delta 11.66(\mathrm{brs}, 1 \mathrm{H}, \mathrm{COOH}), 8.02(\mathrm{~d}, J=8.1 \mathrm{~Hz}, 2 \mathrm{H}), 7.81(\mathrm{~d}, J=8.1 \mathrm{~Hz}, 2 \mathrm{H}), 7.50-7.42$ $(\mathrm{m}, 2 \mathrm{H}), 7.20(\mathrm{~d}, J=8.2 \mathrm{~Hz}, 1 \mathrm{H}), 4.58-4.44(\mathrm{~m}, 2 \mathrm{H}), 4.28(\mathrm{~s}, 2 \mathrm{H}), 3.63(\mathrm{~d}, J=10.7 \mathrm{~Hz}, 2 \mathrm{H}), 3.34-3.17$ (m, 2H). ${ }^{13} \mathrm{C}-\mathrm{NMR}\left(150 \mathrm{MHz}\right.$, DMSO- $\left.d_{6}\right) \delta 166.9,134.3,131.7,131.6,131.0,130.7,130.4,129.6,129.3$, $119.3,57.7,50.8,48.3,39.5,24.4$. ESI-MS $m / z 346.0[\mathrm{M}+\mathrm{H}]^{+}$.

4-((6-methoxy-3,4-dihydroisoquinolin-2(1H)-yl)methyl)benzoic acid (B-4). White solid (yield, 87.7\%). ${ }^{1} \mathrm{H}-\mathrm{NMR}\left(600 \mathrm{MHz}\right.$, DMSO- $\left.d_{6}\right) \delta 7.83(\mathrm{~d}, J=8.0 \mathrm{~Hz}, 2 \mathrm{H}), 7.24(\mathrm{~d}, J=8.0 \mathrm{~Hz}, 2 \mathrm{H}), 6.91-6.87(\mathrm{~m}, 2 \mathrm{H}), 6.61$ $(\mathrm{d}, J=2.3 \mathrm{~Hz}, 1 \mathrm{H}), 3.69(\mathrm{~d}, J=2.2 \mathrm{~Hz}, 5 \mathrm{H}), 3.61(\mathrm{~s}, 2 \mathrm{H}), 2.87(\mathrm{t}, J=5.8 \mathrm{~Hz}, 2 \mathrm{H}), 2.78(\mathrm{t}, J=5.8 \mathrm{~Hz}, 2 \mathrm{H})$. 
${ }^{13}$ C-NMR (150 MHz, DMSO- $\left.d_{6}\right) \delta$ 170.0, 157.4, 139.4, 135.3, 129.0, 127.5, 127.3, 127.0, 126.9, 113.5, 111.8, $61.9,55.0,54.9,50.2,39.5$, 29.0. ESI-MS $m / z 298.1[\mathrm{M}+\mathrm{H}]^{+}$.

4-((6,7-dimethoxy-3,4-dihydroisoquinolin-2(1H)-yl)methyl)benzoic acid (B-5). White solid (yield, 79.9\%). ${ }^{1} \mathrm{H}-\mathrm{NMR}\left(600 \mathrm{MHz}\right.$, DMSO- $\left.d_{6}\right) \delta 7.83(\mathrm{~d}, J=7.8 \mathrm{~Hz}, 2 \mathrm{H}), 7.24(\mathrm{~d}, J=7.8 \mathrm{~Hz}, 2 \mathrm{H}), 6.65(\mathrm{~s}, 1 \mathrm{H}), 6.61$ $(\mathrm{s}, 1 \mathrm{H}), 3.72(\mathrm{~s}, 2 \mathrm{H}), 3.68(\mathrm{~d}, J=5.2 \mathrm{~Hz}, 8 \mathrm{H}), 2.87(\mathrm{t}, J=5.8 \mathrm{~Hz}, 2 \mathrm{H}), 2.71(\mathrm{~d}, J=5.8 \mathrm{~Hz}, 2 \mathrm{H}) .{ }^{13} \mathrm{C}-\mathrm{NMR}$ $\left(150 \mathrm{MHz}, \mathrm{DMSO}-d_{6}\right) \delta 170.0,147.1,146.9,146.8,129.0,127.6,126.7,126.6,125.8,112.4,109.7,61.9,55.5$, $55.4,50.2,39.5,28.3$. ESI-MS $m / z 328.1[\mathrm{M}+\mathrm{H}]^{+}$.

\subsubsection{General Procedure for the Synthesis of 1-30}

The intermediates $\mathbf{B}(0.374 \mathrm{mmol}, 1$ equiv.) were dissolved in $5 \mathrm{~mL}$ DMF in a vial. 2-(7-azabenzotriazol-1-yl)- $N, N, N^{\prime}, N^{\prime}$-tetramethyluronium hexafluorophosphate (HATU) (156.4 mg, $0.411 \mathrm{mmol}, 1.1$ equiv.) were added to the reaction mixture. A series of corresponding amines or others ((4-fluorophenyl)hydrazine, 2-bromophenol and 2-bromobenzenethiol) ( $0.374 \mathrm{mmol}, 1$ equiv.) and DIPEA (193.3 mg, $1.496 \mathrm{mmol}, 4$ equiv.) were made in a separate vial. This solution was added to the reaction mixture drop wise and the reaction mixture was stirred overnight at room temperature. Upon completion of the reaction as detected by TLC, water $(10 \mathrm{~mL})$ was added and the product was extracted with EtOAc $(3 \times 10 \mathrm{~mL})$. The combined organic layers were dried using $\mathrm{MgSO}_{4}$, then concentrated under vacuum, and the residues were purified by flash chromatography using $\mathrm{PE}: \mathrm{EtOAc}=2: 1$ as an eluent to give the target compounds 1-30.

$\mathrm{N}$-(2-(diethylamino)ethyl)-4-((3,4-dihydroisoquinolin-2(1H)-yl)methyl)benzamide (1). Yellow oil (yield 62.5\%). ${ }^{1} \mathrm{H}-\mathrm{NMR}\left(600 \mathrm{MHz}, \mathrm{DMSO}-d_{6}\right) \delta 8.34(\mathrm{t}, J=5.8 \mathrm{~Hz}, 1 \mathrm{H}), 7.80(\mathrm{~d}, J=8.2 \mathrm{~Hz}, 2 \mathrm{H}), 7.44(\mathrm{~d}, J=8.2 \mathrm{~Hz}, 2 \mathrm{H})$, 7.13-7.06 (m, 3H), $6.99(\mathrm{~d}, J=7.3 \mathrm{~Hz}, 1 \mathrm{H}), 3.69(\mathrm{~s}, 2 \mathrm{H}), 3.53(\mathrm{~s}, 2 \mathrm{H}), 3.32-3.29(\mathrm{~m}, 2 \mathrm{H}), 2.81(\mathrm{t}, J=5.8 \mathrm{~Hz}$, $2 \mathrm{H}), 2.67(\mathrm{t}, J=5.8 \mathrm{~Hz}, 2 \mathrm{H}), 2.57-2.50(\mathrm{~m}, 6 \mathrm{H}), 0.97(\mathrm{t}, J=7.1 \mathrm{~Hz}, 6 \mathrm{H}) .{ }^{13} \mathrm{C}-\mathrm{NMR}\left(150 \mathrm{MHz}, \mathrm{DMSO}-d_{6}\right)$ $\delta$ 165.9, 141.7, 134.7, 134.1, 133.4, 128.5, 127.1, 126.3, 126.0, 125.5, 61.4, 55.4, 51.5, 50.3, 46.8, 39.5, 37.5, 28.7, 11.9. ESI-MS $m / z 366.2[\mathrm{M}+\mathrm{H}]^{+}$. HR-ESIMS: $[\mathrm{M}+\mathrm{H}]^{+}$calcd for $\mathrm{C}_{23} \mathrm{H}_{32} \mathrm{~N}_{3} \mathrm{O}^{+} 366.2545$, found 366.2549 .

4-((3,4-dihydroisoquinolin-2(1H)-yl)methyl)-N-(2-(diisopropylamino)ethyl)benzamide (2). Yellow oil (yield $54.7 \%) .{ }^{1} \mathrm{H}-\mathrm{NMR}\left(600 \mathrm{MHz}, \mathrm{DMSO}-\mathrm{d}_{6}\right) \delta 8.34(\mathrm{t}, J=5.8 \mathrm{~Hz}, 1 \mathrm{H}), 7.81(\mathrm{~d}, J=8.2 \mathrm{~Hz}, 2 \mathrm{H}), 7.44(\mathrm{~d}, J=8.2$ $\mathrm{Hz}, 2 \mathrm{H}), 7.12-7.06(\mathrm{~m}, 3 \mathrm{H}), 6.99(\mathrm{~d}, J=7.3 \mathrm{~Hz}, 1 \mathrm{H}), 3.69(\mathrm{~s}, 2 \mathrm{H}), 3.53(\mathrm{~s}, 2 \mathrm{H}), 3.21-3.17(\mathrm{~m}, 2 \mathrm{H}), 3.00-2.94$ $(\mathrm{m}, 2 \mathrm{H}), 2.81(\mathrm{t}, J=5.8 \mathrm{~Hz}, 2 \mathrm{H}), 2.67(\mathrm{t}, J=5.8 \mathrm{~Hz}, 2 \mathrm{H}), 2.53-2.50(\mathrm{~m}, 2 \mathrm{H}), 0.98(\mathrm{~d}, J=6.6 \mathrm{~Hz}, 12 \mathrm{H})$. ${ }^{13} \mathrm{C}-\mathrm{NMR}\left(150 \mathrm{MHz}, \mathrm{DMSO}-d_{6}\right) \delta 165.8,141.6,134.7,134.0,133.4,128.5,127.1,126.3,126.0,125.5,61.4$, 55.4, 50.3, 48.5, 44.2, 41.0, 39.5, 28.7, 20.7. ESI-MS m/z 394.2 [M + H] $]^{+}$. HR-ESIMS: $[\mathrm{M}+\mathrm{H}]^{+}$calcd for $\mathrm{C}_{25} \mathrm{H}_{36} \mathrm{~N}_{3} \mathrm{O}^{+} 394.2858$, found 394.2853.

4-((3,4-dihydroisoquinolin-2(1H)-yl)methyl)- $N$-(2-(dimethylamino)ethyl)benzamide (3). Yellow solid (yield $55.5 \%) .{ }^{1} \mathrm{H}-\mathrm{NMR}\left(600 \mathrm{MHz}, \mathrm{DMSO}-d_{6}\right) \delta 8.35(\mathrm{t}, J=5.7 \mathrm{~Hz}, 1 \mathrm{H}), 7.81(\mathrm{~d}, J=8.2 \mathrm{~Hz}, 2 \mathrm{H}), 7.44(\mathrm{~d}, J=8.2$ $\mathrm{Hz}, 2 \mathrm{H}), 7.13-7.06(\mathrm{~m}, 3 \mathrm{H}), 6.99(\mathrm{~d}, J=7.3 \mathrm{~Hz}, 1 \mathrm{H}), 3.69(\mathrm{~s}, 2 \mathrm{H}), 3.53(\mathrm{~s}, 2 \mathrm{H}), 2.81(\mathrm{t}, J=5.8 \mathrm{~Hz}, 2 \mathrm{H})$, $2.67(\mathrm{t}, J=5.8 \mathrm{~Hz}, 2 \mathrm{H}), 2.50(\mathrm{~d}, J=1.8 \mathrm{~Hz}, 1 \mathrm{H}), 2.50(\mathrm{~d}, J=1.8 \mathrm{~Hz}, 1 \mathrm{H}), 2.41(\mathrm{t}, J=6.8 \mathrm{~Hz}, 2 \mathrm{H}), 2.19(\mathrm{~s}$, 6H). ${ }^{13} \mathrm{C}-\mathrm{NMR}\left(150 \mathrm{MHz}\right.$, DMSO- $\left.d_{6}\right) \delta 166.0,141.7,134.7,134.1,133.3,128.5,127.2,126.4,126.0,125.5$, 61.4, 58.2, 55.4, 50.3, 45.2, 39.5, 37.3, 28.7. ESI-MS m/z 338.2 [M + H] ${ }^{+}$. HR-ESIMS: [M + H] ${ }^{+}$calcd for $\mathrm{C}_{21} \mathrm{H}_{28} \mathrm{~N}_{3} \mathrm{O}^{+} 338.2232$, found 338.2236.

4-((3,4-dihydroisoquinolin-2(1H)-yl)methyl)-N-(2-(ethylamino)ethyl)benzamide (4). Yellow solid (yield 60\%). ${ }^{1} \mathrm{H}-\mathrm{NMR}\left(600 \mathrm{MHz}\right.$, DMSO- $\left.d_{6}\right) \delta 8.39(\mathrm{t}, J=5.6 \mathrm{~Hz}, 1 \mathrm{H}), 7.82(\mathrm{~d}, J=8.2 \mathrm{~Hz}, 2 \mathrm{H}), 7.43(\mathrm{~d}, J=8.2 \mathrm{~Hz}, 2 \mathrm{H})$, 7.13-7.06 (m, 3H), $6.99(\mathrm{~d}, J=7.3 \mathrm{~Hz}, 1 \mathrm{H}), 3.69(\mathrm{~s}, 2 \mathrm{H}), 3.53(\mathrm{~s}, 2 \mathrm{H}), 2.81(\mathrm{t}, J=5.8 \mathrm{~Hz}, 2 \mathrm{H}), 2.69-2.65$ $(\mathrm{m}, 4 \mathrm{H}), 2.56(\mathrm{q}, J=7.1 \mathrm{~Hz}, 2 \mathrm{H}), 2.50(\mathrm{~d}, J=1.8 \mathrm{~Hz}, 2 \mathrm{H}), 2.50(\mathrm{~d}, J=1.8 \mathrm{~Hz}, 1 \mathrm{H}), 1.01(\mathrm{t}, J=7.1 \mathrm{~Hz}, 3 \mathrm{H})$. ${ }^{13} \mathrm{C}-\mathrm{NMR}\left(150 \mathrm{MHz}, \mathrm{DMSO}-d_{6}\right) \delta 166.1,141.7,134.7,134.1,133.4,128.5,128.4,127.2,126.4,126.0,125.5$, 61.4, 55.4, 50.3, 48.5, 43.3, 39.5, 28.7, 15.1. ESI-MS $m / z$ 338.2 [M + H] ${ }^{+}$. HR-ESIMS: $[\mathrm{M}+\mathrm{H}]^{+}$calcd for $\mathrm{C}_{21} \mathrm{H}_{28} \mathrm{~N}_{3} \mathrm{O}^{+}$338.2232, found 338.2213. 
4-((3,4-dihydroisoquinolin-2(1H)-yl)methyl)-N-(2,2-dimethoxyethyl)benzamide (5). Yellow oil (yield 64.3\%). ${ }^{1} \mathrm{H}-\mathrm{NMR}\left(600 \mathrm{MHz}, \mathrm{DMSO}-\mathrm{d}_{6}\right) \delta 8.52((\mathrm{t}, J=5.8 \mathrm{~Hz}, 1 \mathrm{H}), 7.84(\mathrm{~d}, J=8.2 \mathrm{~Hz}, 2 \mathrm{H}), 7.45(\mathrm{~d}, J=8.2 \mathrm{~Hz}$, 2H), 7.13-7.05 (m, 3H), $6.99(\mathrm{~d}, J=7.3 \mathrm{~Hz}, 1 \mathrm{H}), 4.52(\mathrm{t}, J=5.5 \mathrm{~Hz}, 1 \mathrm{H}), 3.69(\mathrm{~s}, 2 \mathrm{H}), 3.53(\mathrm{~s}, 2 \mathrm{H}), 3.38-3.32$ $(\mathrm{m}, 6 \mathrm{H}), 2.81(\mathrm{t}, J=5.8 \mathrm{~Hz}, 2 \mathrm{H}), 2.69-2.65(\mathrm{~m}, 2 \mathrm{H}) .{ }^{13} \mathrm{C}-\mathrm{NMR}\left(150 \mathrm{MHz}, \mathrm{DMSO}-d_{6}\right) \delta 166.2,141.9,134.7$, 134.1, 133.0, 128.5, 128.5, 127.3, 126.4, 126.0, 125.5, 101.9, 61.4, 55.4, 53.2, 53.2, 50.3, 41.1, 39.5, 28.7. ESI-MS m/z 355.1 $[\mathrm{M}+\mathrm{H}]^{+}$. HR-ESIMS: $[\mathrm{M}+\mathrm{H}]^{+}$calcd for $\mathrm{C}_{21} \mathrm{H}_{27} \mathrm{~N}_{2} \mathrm{O}_{3}{ }^{+}$355.2022, found 355.2026.

4-((3,4-dihydroisoquinolin-2(1H)-yl)methyl)-N-(2-morpholinoethyl)benzamide (6). Yellow solid (yield 57\%). ${ }^{1} \mathrm{H}-\mathrm{NMR}\left(600 \mathrm{MHz}, \mathrm{DMSO}-d_{6}\right) \delta 8.37(\mathrm{t}, J=5.6 \mathrm{~Hz}, 1 \mathrm{H}), 7.80(\mathrm{~d}, J=8.2 \mathrm{~Hz}, 2 \mathrm{H}), 7.44(\mathrm{~d}, J=8.2 \mathrm{~Hz}$, $2 \mathrm{H}), 7.13-7.04(\mathrm{~m}, 3 \mathrm{H}), 6.99(\mathrm{~d}, J=7.3 \mathrm{~Hz}, 1 \mathrm{H}), 3.69(\mathrm{~s}, 2 \mathrm{H}), 3.57(\mathrm{t}, J=4.5 \mathrm{~Hz}, 4 \mathrm{H}), 3.53(\mathrm{~s}, 2 \mathrm{H}), 3.38$ $(\mathrm{q}, J=6.6 \mathrm{~Hz}, 2 \mathrm{H}), 2.81(\mathrm{t}, J=5.8 \mathrm{~Hz}, 2 \mathrm{H}), 2.67(\mathrm{t}, J=5.8 \mathrm{~Hz}, 2 \mathrm{H}), 2.50(\mathrm{~s}, 1 \mathrm{H}), 2.46(\mathrm{t}, J=6.9 \mathrm{~Hz}, 2 \mathrm{H})$, $2.41(\mathrm{~s}, 3 \mathrm{H}) .{ }^{13} \mathrm{C}-\mathrm{NMR}\left(150 \mathrm{MHz}, \mathrm{DMSO}-d_{6}\right) \delta 166.0,141.8,134.7,134.1,133.3,128.5,128.5,127.2,126.4$, 126.0, 125.5, 66.2, 61.4, 57.4, 55.4, 53.3, 50.3, 39.5, 36.5, 28.7. ESI-MS m/z 380.2 [M + H] ${ }^{+}$. HR-ESIMS: $[\mathrm{M}+\mathrm{H}]^{+}$calcd for $\mathrm{C}_{23} \mathrm{H}_{30} \mathrm{~N}_{3} \mathrm{O}_{2}{ }^{+} 380.2338$, found 380.2330 .

4-((3,4-dihydroisoquinolin-2(1H)-yl)methyl)-N-(2-(pyrrolidin-1-yl)ethyl)benzamide (7). White solid (yield 60\%). ${ }^{1} \mathrm{H}-\mathrm{NMR}\left(600 \mathrm{MHz}, \mathrm{DMSO}-\mathrm{d}_{6}\right) \delta 8.39(\mathrm{t}, J=5.6 \mathrm{~Hz}, 1 \mathrm{H}), 7.81(\mathrm{~d}, J=8.2 \mathrm{~Hz}, 2 \mathrm{H}), 7.44(\mathrm{~d}, J=8.2$ $\mathrm{Hz}, 2 \mathrm{H}), 7.13-7.06(\mathrm{~m}, 3 \mathrm{H}), 6.99(\mathrm{~d}, J=7.3 \mathrm{~Hz}, 1 \mathrm{H}), 3.69(\mathrm{~s}, 2 \mathrm{H}), 3.53(\mathrm{~s}, 2 \mathrm{H}), 3.37(\mathrm{q}, J=6.8 \mathrm{~Hz}, 2 \mathrm{H}), 2.81$ $(\mathrm{t}, J=5.8 \mathrm{~Hz}, 2 \mathrm{H}), 2.67(\mathrm{t}, J=5.8 \mathrm{~Hz}, 2 \mathrm{H}), 2.56(\mathrm{t}, J=7.0 \mathrm{~Hz}, 2 \mathrm{H}), 2.49-2.45(\mathrm{~m}, 4 \mathrm{H}), 1.67(\mathrm{p}, J=3.1 \mathrm{~Hz}$, $4 \mathrm{H}) .{ }^{13} \mathrm{C}-\mathrm{NMR}\left(150 \mathrm{MHz}, \mathrm{DMSO}-d_{6}\right) \delta 165.9,141.7,134.7,134.1,133.3,128.5,127.2,126.3,126.0,125.5$, 61.4, 55.4, 54.9, 53.7, 50.3, 39.5, 38.6, 28.7, 23.1. ESI-MS m/z 337.1 [M + H] ${ }^{+}$. HR-ESIMS: $[\mathrm{M}+\mathrm{H}]^{+}$calcd for $\mathrm{C}_{23} \mathrm{H}_{30} \mathrm{~N}_{3} \mathrm{O}^{+} 364.2389$, found 364.2365.

4-((3,4-dihydroisoquinolin-2(1H)-yl)methyl)-N-((1-ethylpyrrolidin-2-yl)methyl)benzamide (8). Yellow oil (yield 74.5\%). ${ }^{1} \mathrm{H}-\mathrm{NMR}\left(600 \mathrm{MHz}, \mathrm{DMSO}-d_{6}\right) \delta 8.34(\mathrm{t}, J=5.7 \mathrm{~Hz}, 1 \mathrm{H}), 7.80(\mathrm{~d}, J=8.2 \mathrm{~Hz}, 2 \mathrm{H}), 7.44$ $(\mathrm{d}, J=8.2 \mathrm{~Hz}, 2 \mathrm{H}), 7.13-7.05(\mathrm{~m}, 3 \mathrm{H}), 6.99(\mathrm{~d}, J=7.3 \mathrm{~Hz}, 1 \mathrm{H}), 3.69(\mathrm{~s}, 2 \mathrm{H}), 3.53(\mathrm{~s}, 2 \mathrm{H}), 3.43-3.38(\mathrm{~m}, 1 \mathrm{H})$, 3.08-3.00 (m, 2H), 2.87-2.82 (m, 1H), $2.81(\mathrm{t}, J=5.8 \mathrm{~Hz}, 2 \mathrm{H}), 2.67(\mathrm{t}, J=5.8 \mathrm{~Hz}, 2 \mathrm{H}), 2.57(\mathrm{~m}, 1 \mathrm{H}), 2.27(\mathrm{~m}$, 1H), 2.15-2.08 (m, 1H), $1.77(\mathrm{~m}, 1 \mathrm{H}), 1.67-1.55(\mathrm{~m}, 3 \mathrm{H}), 1.04(\mathrm{t}, J=7.2 \mathrm{~Hz}, 3 \mathrm{H}) .{ }^{13} \mathrm{C}-\mathrm{NMR}(150 \mathrm{MHz}$, DMSO- $\left.d_{6}\right) \delta 166.1,141.7,134.7,134.1,133.4,128.5,127.2,126.3,126.0,125.5,62.7,61.4,55.4,53.3,50.3$, 48.2, 43.2, 39.5, 28.7, 28.6, 22.4, 13.9. ESI-MS $m / z 378.2[\mathrm{M}+\mathrm{H}]^{+}$. HR-ESIMS: $[\mathrm{M}+\mathrm{H}]^{+}$calcd for $\mathrm{C}_{24} \mathrm{H}_{32} \mathrm{~N}_{3} \mathrm{O}^{+} 378.2545$, found 378.2563.

(4-((3,4-dihydroisoquinolin-2(1H)-yl)methyl)phenyl)(pyrrolidin-1-yl)methanone (9). Yellow oil (yield 74\%). ${ }^{1} \mathrm{H}-\mathrm{NMR}\left(600 \mathrm{MHz}, \mathrm{DMSO}-d_{6}\right) \delta 7.48(\mathrm{~d}, J=8.1 \mathrm{~Hz}, 2 \mathrm{H}), 7.41(\mathrm{~d}, J=8.1 \mathrm{~Hz}, 2 \mathrm{H}), 7.09(\mathrm{~m}, 3 \mathrm{H}), 6.99$ $(\mathrm{d}, J=7.3 \mathrm{~Hz}, 1 \mathrm{H}), 3.67(\mathrm{~s}, 2 \mathrm{H}), 3.54(\mathrm{~s}, 2 \mathrm{H}), 3.48-3.39(\mathrm{~m}, 4 \mathrm{H}), 2.81(\mathrm{t}, J=5.8 \mathrm{~Hz}, 2 \mathrm{H}), 2.68(\mathrm{t}, J=5.8 \mathrm{~Hz}$, 2H), 1.88-1.83 (m, 2H), 1.82-1.77 (m, 2H). ${ }^{13} \mathrm{C}-\mathrm{NMR}\left(150 \mathrm{MHz}, \mathrm{DMSO}-d_{6}\right) \delta 168.2,140.2,135.9,134.7$, $134.1,128.5,128.4,127.1,126.4,126.0,125.5,61.5,55.4,50.3,49.0,45.9,39.5,28.7,26.0,23.9$. ESI-MS $m / z$ $321.1[\mathrm{M}+\mathrm{H}]^{+}$. HR-ESIMS: $[\mathrm{M}+\mathrm{H}]^{+}$calcd for $\mathrm{C}_{21} \mathrm{H}_{25} \mathrm{~N}_{2} \mathrm{O}^{+} 321.1967$, found 321.1997.

(4-((3,4-dihydroisoquinolin-2(1H)-yl)methyl)phenyl)(morpholino)methanone (10). Yellow oil (yield 68\%). ${ }^{1} \mathrm{H}-\mathrm{NMR}\left(600 \mathrm{MHz}, \mathrm{DMSO}-\mathrm{d}_{6}\right) \delta 7.43(\mathrm{~d}, J=8.1 \mathrm{~Hz}, 2 \mathrm{H}), 7.38(\mathrm{~d}, J=8.1 \mathrm{~Hz}, 2 \mathrm{H}), 7.13-7.06(\mathrm{~m}, 3 \mathrm{H})$, $7.00(\mathrm{~d}, J=7.2 \mathrm{~Hz}, 1 \mathrm{H}), 3.68(\mathrm{~s}, 2 \mathrm{H}), 3.59(\mathrm{~s}, 4 \mathrm{H}), 3.55(\mathrm{~s}, 2 \mathrm{H}), 2.82(\mathrm{t}, J=5.8 \mathrm{~Hz}, 2 \mathrm{H}), 2.68(\mathrm{t}, J=5.8$ $\mathrm{Hz}, 2 \mathrm{H}) .{ }^{13} \mathrm{C}-\mathrm{NMR}(150 \mathrm{MHz}$, DMSO-d $) \delta 169.1,140.1,134.7,134.2,134.1,128.6,128.5,127.1,126.4$, 126.0, 125.5, 66.1, 61.4, 55.4, 50.2, 39.5, 28.7. ESI-MS m/z 337.1 [M + H] $]^{+}$. HR-ESIMS: $[\mathrm{M}+\mathrm{H}]^{+}$calcd for $\mathrm{C}_{21} \mathrm{H}_{25} \mathrm{~N}_{2} \mathrm{O}_{2}+337.1916$, found 337.1909.

(4-((6-bromo-3,4-dihydroisoquinolin-2(1H)-yl)methyl)phenyl)(pyrrolidin-1-yl)methanone (11). Yellow oil (yield 64\%). ${ }^{1} \mathrm{H}-\mathrm{NMR}\left(600 \mathrm{MHz}, \mathrm{DMSO}-\mathrm{d}_{6}\right) \delta 7.48(\mathrm{~d}, J=8.1 \mathrm{~Hz}, 2 \mathrm{H}), 7.40(\mathrm{~d}, J=8.1 \mathrm{~Hz}, 2 \mathrm{H}), 7.32$ $(\mathrm{d}, J=2.1 \mathrm{~Hz}, 1 \mathrm{H}), 7.26(\mathrm{dd}, J=8.2,2.1 \mathrm{~Hz}, 1 \mathrm{H}), 6.99(\mathrm{~d}, J=8.2 \mathrm{~Hz}, 1 \mathrm{H}), 3.68(\mathrm{~s}, 2 \mathrm{H}), 3.50(\mathrm{~s}, 2 \mathrm{H}), 3.45$ $(\mathrm{t}, J=6.7 \mathrm{~Hz}, 2 \mathrm{H}), 3.38(\mathrm{t}, J=6.7 \mathrm{~Hz}, 2 \mathrm{H}), 2.82(\mathrm{t}, J=5.8 \mathrm{~Hz}, 2 \mathrm{H}), 2.66(\mathrm{t}, J=5.8 \mathrm{~Hz}, 2 \mathrm{H}), 1.88-1.83$ $(\mathrm{m}, 2 \mathrm{H}), 1.82-1.77$ (m. 2H). ${ }^{13} \mathrm{C}-\mathrm{NMR}\left(150 \mathrm{MHz}, \mathrm{DMSO}-d_{6}\right) \delta 168.1,140.0,137.1,135.9,134.2,131.0,128.6$, 128.4, 128.3, 127.1, 118.8, 61.2, 54.8, 49.7, 49.0, 45.9, 39.5, 28.4, 26.0, 23.9. ESI-MS m/z $399.0[\mathrm{M}+\mathrm{H}]^{+}$. HR-ESIMS: $[\mathrm{M}+\mathrm{H}]^{+}$calcd for $\mathrm{C}_{21} \mathrm{H}_{24} \mathrm{BrN}_{2} \mathrm{O}^{+} 399.1072$, found 399.1056. 
(4-((7-bromo-3,4-dihydroisoquinolin-2(1H)-yl)methyl)phenyl)(pyrrolidin-1-yl)methanone (12). Yellow oil (yield 65\%). ${ }^{1} \mathrm{H}-\mathrm{NMR}\left(600 \mathrm{MHz}, \mathrm{DMSO}-\mathrm{d}_{6}\right) \delta 7.48(\mathrm{~d}, J=7.8 \mathrm{~Hz}, 2 \mathrm{H}), 7.40(\mathrm{~d}, J=7.8 \mathrm{~Hz}, 2 \mathrm{H}), 7.29$ $(\mathrm{d}, J=8.2 \mathrm{~Hz}, 1 \mathrm{H}), 7.25(\mathrm{~s}, 1 \mathrm{H}), 7.07(\mathrm{~d}, J=8.2 \mathrm{~Hz}, 1 \mathrm{H}), 3.67(\mathrm{~s}, 2 \mathrm{H}), 3.55(\mathrm{~s}, 2 \mathrm{H}), 3.46(\mathrm{t}, J=6.7 \mathrm{~Hz}$, $2 \mathrm{H}), 3.39(\mathrm{t}, J=6.7 \mathrm{~Hz}, 2 \mathrm{H}), 2.77(\mathrm{t}, J=5.7 \mathrm{~Hz}, 2 \mathrm{H}), 2.67(\mathrm{t}, J=5.7 \mathrm{~Hz}, 2 \mathrm{H}), 1.89-1.83(\mathrm{~m}, 2 \mathrm{H}), 1.82-1.77$ $(\mathrm{m}, 2 \mathrm{H}) .{ }^{13} \mathrm{C}-\mathrm{NMR}\left(150 \mathrm{MHz}, \mathrm{DMSO}-d_{6}\right) \delta 168.1,140.0,137.5,135.9,133.6,130.7,129.0,128.8,128.3$, 127.1, 118.3, 61.1, 54.8, 49.8, 48.9, 45.9, 39.5, 28.1, 26.0, 23.9. ESI-MS m/z 399.0 [M + H] ${ }^{+}$. HR-ESIMS: $[\mathrm{M}+\mathrm{H}]^{+}$calcd for $\mathrm{C}_{21} \mathrm{H}_{24} \mathrm{BrN}_{2} \mathrm{O}^{+} 399.1072$, found 399.1062 .

(4-((6-methoxy-3,4-dihydroisoquinolin-2(1H)-yl)methyl)phenyl)(pyrrolidin-1-yl)methanone (13). Brown oil (yield 71\%). ${ }^{1} \mathrm{H}-\mathrm{NMR}\left(600 \mathrm{MHz}, \mathrm{DMSO}-\mathrm{d}_{6}\right) \delta 7.48(\mathrm{~d}, J=8.0 \mathrm{~Hz}, 2 \mathrm{H}), 7.40(\mathrm{~d}, J=8.0 \mathrm{~Hz}, 2 \mathrm{H}), 6.91$ $(\mathrm{d}, J=9.2 \mathrm{~Hz}, 1 \mathrm{H}), 6.67(\mathrm{~d}, J=6.6 \mathrm{~Hz}, 2 \mathrm{H}), 3.69(\mathrm{~s}, 3 \mathrm{H}), 3.66(\mathrm{~s}, 2 \mathrm{H}), 3.48-3.44(\mathrm{~m}, 4 \mathrm{H}), 3.39(\mathrm{t}, J=6.5$ $\mathrm{Hz}, 2 \mathrm{H}), 2.79(\mathrm{t}, J=5.8 \mathrm{~Hz}, 2 \mathrm{H}), 2.65(\mathrm{t}, J=5.8 \mathrm{~Hz}, 2 \mathrm{H}), 1.88-1.83(\mathrm{~m}, 2 \mathrm{H}), 1.82-1.77(\mathrm{~m}, 2 \mathrm{H}) .{ }^{13} \mathrm{C}-\mathrm{NMR}$ $\left(150 \mathrm{MHz}, \mathrm{DMSO}-d_{6}\right) \delta 168.1,157.5,140.2,135.9,135.2,128.3,127.3,127.1,126.8,113.0,111.9,61.5$, $54.9,50.2,48.9,45.9,39.5,29.0,26.0,23.9$. ESI-MS m/z 351.1 [M + H] ${ }^{+}$. HR-ESIMS: $[\mathrm{M}+\mathrm{H}]^{+}$calcd for $\mathrm{C}_{22} \mathrm{H}_{27} \mathrm{~N}_{2} \mathrm{O}_{2}{ }^{+} 351.2073$, found 351.2079.

(4-((6,7-dimethoxy-3,4-dihydroisoquinolin-2(1H)-yl)methyl)phenyl)(pyrrolidin-1-yl)methanone (14). Yellow oil (yield 60.3\%). ${ }^{1} \mathrm{H}-\mathrm{NMR}\left(600 \mathrm{MHz}, \mathrm{DMSO}-\mathrm{d}_{6}\right) \delta 7.48(\mathrm{~d}, J=8.0 \mathrm{~Hz}, 2 \mathrm{H}), 7.40(\mathrm{~d}, J=8.0 \mathrm{~Hz}, 2 \mathrm{H})$, $6.66(\mathrm{~s}, 1 \mathrm{H}), 6.58(\mathrm{~s}, 1 \mathrm{H}), 3.69(\mathrm{~s}, 3 \mathrm{H}), 3.66(\mathrm{~s}, 5 \mathrm{H}), 3.46(\mathrm{t}, J=6.9 \mathrm{~Hz}, 2 \mathrm{H}), 3.43(\mathrm{~s}, 2 \mathrm{H}), 3.39(\mathrm{t}, J=6.5$ $\mathrm{Hz}, 2 \mathrm{H}), 2.73(\mathrm{t}, J=5.6 \mathrm{~Hz}, 2 \mathrm{H}), 2.66(\mathrm{t}, J=5.6 \mathrm{~Hz}, 2 \mathrm{H}), 1.88-1.84(\mathrm{~m}, 2 \mathrm{H}), 1.82-1.77(\mathrm{~m}, 2 \mathrm{H}) .{ }^{13} \mathrm{C}-\mathrm{NMR}$ $\left(151 \mathrm{MHz}, \mathrm{DMSO}-d_{6}\right) \delta 168.1,147.2,146.9,140.3,135.9,128.4,127.1,126.4,125.7,111.8,109.9,61.6,55.5$, $55.4,55.0,50.6,49.0,45.9,28.3,26.0,23.9$. ESI-MS m/z 381.1 [M + H] ${ }^{+}$. HR-ESIMS: $[\mathrm{M}+\mathrm{H}]^{+}$calcd for $^{\circ}$ $\mathrm{C}_{23} \mathrm{H}_{29} \mathrm{~N}_{2} \mathrm{O}_{3}+381.2178$, found 381.2170 .

4-((3,4-dihydroisoquinolin-2(1H)-yl)methyl)-N-phenylbenzamide (15). White solid (yield 56\%). ${ }^{1} \mathrm{H}-\mathrm{NMR}$ (600 MHz, DMSO-d $\left.)_{6}\right) \delta 10.22$ (brs, 1H, NH), $7.93(\mathrm{~d}, J=8.2 \mathrm{~Hz}, 2 \mathrm{H}), 7.77(\mathrm{~d}, J=7.6 \mathrm{~Hz}, 2 \mathrm{H}), 7.52$ $(\mathrm{d}, J=8.2 \mathrm{~Hz}, 2 \mathrm{H}), 7.37-7.32(\mathrm{~m}, 2 \mathrm{H}), 7.14-7.06(\mathrm{~m}, 4 \mathrm{H}), 7.01(\mathrm{~s}, 1 \mathrm{H}), 3.73(\mathrm{~s}, 2 \mathrm{H}), 3.56(\mathrm{~s}, 2 \mathrm{H}), 2.83(\mathrm{t}$, $J=5.9 \mathrm{~Hz}, 2 \mathrm{H}), 2.70(\mathrm{t}, J=5.9 \mathrm{~Hz}, 2 \mathrm{H}) .{ }^{13} \mathrm{C}-\mathrm{NMR}\left(150 \mathrm{MHz}, \mathrm{DMSO}-d_{6}\right) \delta 165.5,142.4,139.2,134.7,134.1$, $133.7,128.6,128.6,128.5,127.8,126.4,126.1,125.5,123.7,120.4,61.4,55.5,50.3,39.5,28.7$. ESI-MS m/z $343.1[\mathrm{M}+\mathrm{H}]^{+}$. HR-ESIMS: $[\mathrm{M}+\mathrm{H}]^{+}$calcd for $\mathrm{C}_{23} \mathrm{H}_{23} \mathrm{~N}_{2} \mathrm{O}^{+} 343.1810$, found 343.1827.

4-((3,4-dihydroisoquinolin-2(1H)-yl)methyl)-N-(2-fluorophenyl)benzamide (16a). Yellow oil (yield 37\%). ${ }^{1} \mathrm{H}-\mathrm{NMR}\left(600 \mathrm{MHz}, \mathrm{DMSO}-d_{6}\right) \delta 10.09$ (brs, $\left.1 \mathrm{H}, \mathrm{NH}\right), 7.96(\mathrm{~d}, J=8.2 \mathrm{~Hz}, 2 \mathrm{H}), 7.61-7.57(\mathrm{~m}, 1 \mathrm{H}), 7.52$ $(\mathrm{d}, J=8.2 \mathrm{~Hz}, 2 \mathrm{H}), 7.32-7.25(\mathrm{~m}, 2 \mathrm{H}), 7.24-7.2(\mathrm{~m}, 1 \mathrm{H}), 7.15-7.06(\mathrm{~m}, 3 \mathrm{H}), 7.01(\mathrm{~d}, J=7.3 \mathrm{~Hz}, 1 \mathrm{H}), 3.74$ $(\mathrm{s}, 2 \mathrm{H}), 3.56(\mathrm{~s}, 2 \mathrm{H}), 2.83(\mathrm{t}, J=5.8 \mathrm{~Hz}, 2 \mathrm{H}), 2.70(\mathrm{t}, J=5.8 \mathrm{~Hz}, 2 \mathrm{H}) .{ }^{13} \mathrm{C}-\mathrm{NMR}\left(150 \mathrm{MHz}, \mathrm{DMSO}-d_{6}\right) \delta$ $165.3,156.7,155.0,142.7,134.7,134.1,132.7,128.6,128.5,127.9,127.2,126.4,126.0,125.5,124.3,124.3$, $115.9,115.8,61.4,55.4,50.3,39.5,28.7$. ESI-MS $m / z 361.0[\mathrm{M}+\mathrm{H}]^{+}$. HR-ESIMS: $[\mathrm{M}+\mathrm{H}]^{+}$calcd for $\mathrm{C}_{23} \mathrm{H}_{22} \mathrm{FN}_{2} \mathrm{O}^{+}$361.1716, found 361.1757.

4-((3,4-dihydroisoquinolin-2(1H)-yl)methyl)-N-(3-fluorophenyl)benzamide (17). Yellow oil (yield 71.6\%). ${ }^{1} \mathrm{H}-\mathrm{NMR}\left(600 \mathrm{MHz}, \mathrm{DMSO}-d_{6}\right) \delta 10.40$ (brs, $\left.1 \mathrm{H}, \mathrm{NH}\right), 7.93(\mathrm{~d}, J=8.2 \mathrm{~Hz}, 2 \mathrm{H}), 7.78-7.73(\mathrm{~m}, 1 \mathrm{H}), 7.56$ $(\mathrm{dd}, J=8.1,1.9 \mathrm{~Hz}, 1 \mathrm{H}), 7.53(\mathrm{~d}, J=8.1 \mathrm{~Hz}, 2 \mathrm{H}), 7.38(\mathrm{q}, J=8.1 \mathrm{~Hz}, 1 \mathrm{H}), 7.13-7.07(\mathrm{~m}, 3 \mathrm{H}), 7.00(\mathrm{~d}, J=7.2$ $\mathrm{Hz}, 1 \mathrm{H}), 6.96-6.89(\mathrm{~m}, 1 \mathrm{H}), 3.73(\mathrm{~s}, 2 \mathrm{H}), 3.56(\mathrm{~s}, 2 \mathrm{H}), 2.83(\mathrm{t}, J=5.7 \mathrm{~Hz}, 2 \mathrm{H}), 2.71-2.68(\mathrm{~m}, 2 \mathrm{H}) .{ }^{13} \mathrm{C}-\mathrm{NMR}$ $\left(150 \mathrm{MHz}, \mathrm{DMSO}-d_{6}\right) \delta 165.7,162.9,161.3,142.7,141.1,141.0,134.7,134.1,133.4,130.3,130.2,128.6$,

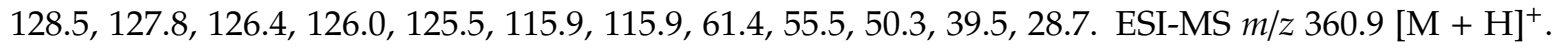
HR-ESIMS: $[\mathrm{M}+\mathrm{H}]^{+}$calcd for $\mathrm{C}_{23} \mathrm{H}_{22} \mathrm{FN}_{2} \mathrm{O}^{+} 361.1716$, found 361.1722 .

4-((3,4-dihydroisoquinolin-2(1H)-yl)methyl)-N-(4-fluorophenyl)benzamide (18). White solid (yield 60\%). ${ }^{1} \mathrm{H}-\mathrm{NMR}\left(600 \mathrm{MHz}, \mathrm{DMSO}-d_{6}\right) \delta 10.30$ (brs, 1H, NH), 7.93 (d, J = 8.2 Hz, 2H), $7.79(\mathrm{~m}, 2 \mathrm{H}), 7.52$ $(\mathrm{d}, J=8.2 \mathrm{~Hz}, 2 \mathrm{H}), 7.22-7.17(\mathrm{~m}, 2 \mathrm{H}), 7.10(\mathrm{~m}, 3 \mathrm{H}), 7.00(\mathrm{~d}, J=7.3 \mathrm{~Hz}, 1 \mathrm{H}), 3.73(\mathrm{~s}, 2 \mathrm{H}), 3.56(\mathrm{~s}, 2 \mathrm{H}), 2.83$ $(\mathrm{t}, J=5.8 \mathrm{~Hz}, 2 \mathrm{H}), 2.69(\mathrm{t}, J=5.8 \mathrm{~Hz}, 2 \mathrm{H}) .{ }^{13} \mathrm{C}-\mathrm{NMR}\left(150 \mathrm{MHz}, \mathrm{DMSO}-d_{6}\right) \delta 165.4,159.1,157.5,142.5$, 135.6, 135.6, 134.7, 134.1, 133.6, 128.6, 128.5, 127.8, 126.4, 126.1, 125.6, 122.2, 122.1, 115.3, 115.2, 61.4, 55.5, 50.3, 39.5, 28.7. ESI-MS $m / z 361.0[\mathrm{M}+\mathrm{H}]^{+}$. HR-ESIMS: $[\mathrm{M}+\mathrm{H}]^{+}$calcd for $\mathrm{C}_{23} \mathrm{H}_{22} \mathrm{FN}_{2} \mathrm{O}^{+} 361.1716$, found 361.1707 . 
4-((3,4-dihydroisoquinolin-2(1H)-yl)methyl)- $N$-(4-fluorobenzyl)benzamide (19). Yellow solid (yield 61.2\%). ${ }^{1} \mathrm{H}-\mathrm{NMR}\left(600 \mathrm{MHz}\right.$, DMSO- $\left.d_{6}\right) \delta 9.03(\mathrm{t}, J=6.0 \mathrm{~Hz}, 1 \mathrm{H}), 7.87(\mathrm{~d}, J=8.3 \mathrm{~Hz}, 2 \mathrm{H}), 7.46(\mathrm{~d}, J=8.3 \mathrm{~Hz}, 2 \mathrm{H})$, 7.40-7.31 (m, 2H), 7.17-7.13 (m, 2H), 7.12-7.05 (m, 3H), $6.99(\mathrm{~d}, J=7.3 \mathrm{~Hz}, 1 \mathrm{H}), 4.46(\mathrm{~d}, J=6.0 \mathrm{~Hz}, 2 \mathrm{H})$, $3.69(\mathrm{~s}, 2 \mathrm{H}), 3.54(\mathrm{~s}, 2 \mathrm{H}), 2.81(\mathrm{t}, J=5.8 \mathrm{~Hz}, 2 \mathrm{H}), 2.67(\mathrm{t}, J=5.8 \mathrm{~Hz}, 2 \mathrm{H}) .{ }^{13} \mathrm{C}-\mathrm{NMR}\left(150 \mathrm{MHz}, \mathrm{DMSO}-d_{6}\right)$ $\delta 166.1,161.9,160.3,142.0,135.9,135.9,134.7,134.0,133.0,129.2,129.2,128.5,128.5,127.3,126.3,126.0$, 125.5, 115.0, 114.9, 61.4, 55.4, 50.3, 41.9, 39.5, 28.7. ESI-MS m/z 375.1 [M + H] ${ }^{+}$. HR-ESIMS: $[\mathrm{M}+\mathrm{H}]^{+}$ calcd for $\mathrm{C}_{24} \mathrm{H}_{24} \mathrm{FN}_{2} \mathrm{O}^{+} 375.1873$, found 375.1890 .

4-((3,4-dihydroisoquinolin-2(1H)-yl)methyl)-N2019-(4-fluorophenyl)benzohydrazide (20). Yellow solid (yield 50\%). ${ }^{1} \mathrm{H}-\mathrm{NMR}\left(600 \mathrm{MHz}, \mathrm{DMSO}-d_{6}\right) \delta 10.39(\mathrm{~d}, J=3.0 \mathrm{~Hz}, 1 \mathrm{H}), 7.92(\mathrm{~d}, J=3.0 \mathrm{~Hz}, 1 \mathrm{H})$, $7.90(\mathrm{~d}, J=8.1 \mathrm{~Hz}, 2 \mathrm{H}), 7.49(\mathrm{~d}, J=8.1 \mathrm{~Hz}, 2 \mathrm{H}), 7.16-7.05(\mathrm{~m}, 3 \mathrm{H}), 7.00(\mathrm{t}, J=8.3 \mathrm{~Hz}, 3 \mathrm{H}), 6.84-6.73$ $(\mathrm{m}, 2 \mathrm{H}), 3.71(\mathrm{~s}, 2 \mathrm{H}), 3.55(\mathrm{~s}, 2 \mathrm{H}), 2.82(\mathrm{t}, J=5.8 \mathrm{~Hz}, 2 \mathrm{H}), 2.69(\mathrm{t}, J=5.8 \mathrm{~Hz}, 2 \mathrm{H}) .{ }^{13} \mathrm{C}-\mathrm{NMR}(150 \mathrm{MHz}$, DMSO- $\left.d_{6}\right) \delta 166.3,156.7,155.1,146.2,142.5,134.7,134.1,131.7,128.7,128.5,127.4,126.4,126.1,125.6$, $115.3,115.2,113.6,113.5,61.4,55.5,50.3,39.5,28.7$. ESI-MS $m / z 376.0[\mathrm{M}+\mathrm{H}]^{+}$. HR-ESIMS: $[\mathrm{M}+\mathrm{H}]^{+}$ calcd for $\mathrm{C}_{23} \mathrm{H}_{23} \mathrm{FN}_{3} \mathrm{O}^{+} 376.1825$, found 376.1845 .

$\mathrm{N}$-(4-chloro-2-fluorophenyl)-4-((3,4-dihydroisoquinolin-2(1H)-yl)methyl)benzamide (21). Yellow oil (yield 34\%). ${ }^{1} \mathrm{H}-\mathrm{NMR}\left(600 \mathrm{MHz}\right.$, DMSO- $\left.d_{6}\right) \delta 8.71(\mathrm{dd}, J=4.3,1.2 \mathrm{~Hz}, 1 \mathrm{H}), 8.50(\mathrm{dd}, J=8.3,1.2 \mathrm{~Hz}, 1 \mathrm{H}), 7.92$ $(\mathrm{d}, J=8.3 \mathrm{~Hz}, 2 \mathrm{H}), 7.50(\mathrm{~d}, J=8.3 \mathrm{~Hz}, 2 \mathrm{H}), 7.49-7.47(\mathrm{~m}, 1 \mathrm{H}), 7.13-7.07(\mathrm{~m}, 3 \mathrm{H}), 7.00(\mathrm{~d}, J=7.3 \mathrm{~Hz}, 1 \mathrm{H})$, $3.76(\mathrm{~s}, 2 \mathrm{H}), 3.58(\mathrm{~s}, 2 \mathrm{H}), 2.84(\mathrm{t}, J=5.8 \mathrm{~Hz}, 2 \mathrm{H}), 2.73(\mathrm{t}, J=5.8 \mathrm{~Hz}, 2 \mathrm{H}) .{ }^{13} \mathrm{C}-\mathrm{NMR}\left(150 \mathrm{MHz}, \mathrm{DMSO}-d_{6}\right)$ $\delta$ 167.2, 150.7, 139.6, 134.6, 133.9, 129.6, 129.4, 128.8, 128.7, 128.5, 126.4, 126.1, 125.6, 120.5, 61.2, 55.3, 50.2, 39.5, 28.5. ESI-MS m/z 395.0 [M + H] $]^{+}$. HR-ESIMS: $[\mathrm{M}+\mathrm{H}]^{+}$calcd for $\mathrm{C}_{23} \mathrm{H}_{21} \mathrm{ClFN}_{2} \mathrm{O}^{+} 395.1326$, found 395.1301 .

N-(4-bromo-2-fluorophenyl)-4-((3,4-dihydroisoquinolin-2(1H)-yl)methyl)benzamide (22). White solid (yield $40 \%) .{ }^{1} \mathrm{H}-\mathrm{NMR}\left(600 \mathrm{MHz}, \mathrm{DMSO}-d_{6}\right) \delta 10.19$ (brs, $\left.1 \mathrm{H}, \mathrm{NH}\right), 7.95(\mathrm{~d}, J=8.2 \mathrm{~Hz}, 2 \mathrm{H}), 7.66(\mathrm{dd}, J=10.0$, $2.1 \mathrm{~Hz}, 1 \mathrm{H}), 7.58(\mathrm{t}, J=8.4 \mathrm{~Hz}, 1 \mathrm{H}), 7.52(\mathrm{~d}, J=8.2 \mathrm{~Hz}, 2 \mathrm{H}), 7.46-7.43(\mathrm{~m}, 1 \mathrm{H}), 7.13-7.07(\mathrm{~m}, 3 \mathrm{H}), 7.00$ $(\mathrm{d}, J=7.3 \mathrm{~Hz}, 1 \mathrm{H}), 3.73(\mathrm{~s}, 2 \mathrm{H}), 3.56(\mathrm{~s}, 2 \mathrm{H}), 2.83(\mathrm{t}, J=5.8 \mathrm{~Hz}, 2 \mathrm{H}), 2.70(\mathrm{t}, J=5.8 \mathrm{~Hz}, 2 \mathrm{H}) .{ }^{13} \mathrm{C}-\mathrm{NMR}$ $\left(150 \mathrm{MHz}, \mathrm{DMSO}-d_{6}\right) \delta 165.3,156.5,154.8,142.9,134.7,134.1,132.4,128.7,128.5,128.4,128.0,127.5$, 126.4, 126.1, 125.6, 119.4, 119.2, 117.6, 117.6, 61.4, 55.5, 50.3, 39.5, 28.7. ESI-MS $m / z 439.0[\mathrm{M}+\mathrm{H}]^{+}$. HR-ESIMS: $[\mathrm{M}+\mathrm{H}]^{+}$calcd for $\mathrm{C}_{23} \mathrm{H}_{21} \mathrm{BrFN}_{2} \mathrm{O}^{+} 439.0821$, found 439.0802 .

$N$-(2-bromophenyl)-4-((3,4-dihydroisoquinolin-2(1H)-yl)methyl)benzamide (23). Yellow oil (yield 51\%). ${ }^{1} \mathrm{H}-\mathrm{NMR}\left(600 \mathrm{MHz}, \mathrm{DMSO}-d_{6}\right) \delta 8.69(\mathrm{dd}, J=4.4,1.4 \mathrm{~Hz}, 1 \mathrm{H}), 8.48(\mathrm{dd}, J=8.4,1.4 \mathrm{~Hz}, 1 \mathrm{H}), 7.93$ $(\mathrm{d}, J=8.2 \mathrm{~Hz}, 2 \mathrm{H}), 7.50(\mathrm{~d}, J=8.2 \mathrm{~Hz}, 2 \mathrm{H}), 7.46(\mathrm{dd}, J=8.4,4.4 \mathrm{~Hz}, 1 \mathrm{H}), 7.14-7.07(\mathrm{~m}, 3 \mathrm{H}), 7.01(\mathrm{~d}, J=7.3$ $\mathrm{Hz}, 1 \mathrm{H}), 3.79(\mathrm{~s}, 2 \mathrm{H}), 3.61(\mathrm{~s}, 2 \mathrm{H}), 2.84(\mathrm{t}, J=5.8 \mathrm{~Hz}, 2 \mathrm{H}), 2.75(\mathrm{t}, J=5.8 \mathrm{~Hz}, 2 \mathrm{H}) .{ }^{13} \mathrm{C}-\mathrm{NMR}(150 \mathrm{MHz}$, DMSO- $\left.d_{6}\right) \delta 167.21,150.59,139.59,134.60,134.10,133.79,129.74,129.39,128.92,128.61,128.46,126.38$, 126.16, 125.58, 120.44, 61.10, 55.14, 50.19, 39.5, 28.35. ESI-MS m/z $421.0[\mathrm{M}+\mathrm{H}]^{+}$. HR-ESIMS: $[\mathrm{M}+\mathrm{H}]^{+}$ calcd for $\mathrm{C}_{23} \mathrm{H}_{22} \mathrm{BrN}_{2} \mathrm{O}^{+}$421.0916, found 421.0910.

N-(3-bromophenyl)-4-((3,4-dihydroisoquinolin-2(1H)-yl)methyl)benzamide (24). Yellow oil (yield 61.1\%). ${ }^{1} \mathrm{H}-\mathrm{NMR}\left(600 \mathrm{MHz}\right.$, DMSO- $\left.d_{6}\right) \delta 10.38$ (brs, $\left.1 \mathrm{H}, \mathrm{NH}\right), 8.12(\mathrm{~d}, J=1.9 \mathrm{~Hz}, 1 \mathrm{H}), 7.95-7.92(\mathrm{~m}, 2 \mathrm{H}), 7.76$ $(\mathrm{d}, J=7.9 \mathrm{~Hz}, 1 \mathrm{H}), 7.53(\mathrm{~d}, J=8.0 \mathrm{~Hz}, 2 \mathrm{H}), 7.31(\mathrm{~m}, 2 \mathrm{H}), 7.13-7.07(\mathrm{~m}, 3 \mathrm{H}), 7.00(\mathrm{~d}, J=7.2 \mathrm{~Hz}, 1 \mathrm{H}), 3.73$ $(\mathrm{s}, 2 \mathrm{H}), 3.57(\mathrm{~s}, 2 \mathrm{H}), 2.83(\mathrm{t}, J=5.7 \mathrm{~Hz}, 2 \mathrm{H}), 2.70(\mathrm{~m}, 2 \mathrm{H}) .{ }^{13} \mathrm{C}-\mathrm{NMR}\left(150 \mathrm{MHz}\right.$, DMSO- $\left.d_{6}\right) \delta 165.7,142.7$, 140.9, 134.7, 134.1, 133.3, 130.6, 128.7, 128.6, 128.5, 127.8, 126.4, 126.2, 125.5, 122.5, 121.4, 119.0, 61.4, 55.5, 50.3, 39.5, 28.7. ESI-MS $m / z 421.0[\mathrm{M}+\mathrm{H}]^{+}$. HR-ESIMS: $[\mathrm{M}+\mathrm{H}]^{+}$calcd for $\mathrm{C}_{23} \mathrm{H}_{22} \mathrm{BrN}_{2} \mathrm{O}^{+} 421.0916$, found 421.0915 .

N-(4-bromophenyl)-4-((3,4-dihydroisoquinolin-2(1H)-yl)methyl)benzamide (25). White solid (yield 38\%). ${ }^{1} \mathrm{H}-\mathrm{NMR}\left(600 \mathrm{MHz}\right.$, DMSO- $\left.d_{6}\right) \delta 10.36(\mathrm{brs}, 1 \mathrm{H}, \mathrm{NH}), 7.93(\mathrm{~d}, J=8.2 \mathrm{~Hz}, 2 \mathrm{H}), 7.80-7.73(\mathrm{~m}, 2 \mathrm{H}), 7.53$ $(\mathrm{m}, 4 \mathrm{H}), 7.14-7.07(\mathrm{~m}, 3 \mathrm{H}), 7.00(\mathrm{~d}, J=7.3 \mathrm{~Hz}, 1 \mathrm{H}), 3.73(\mathrm{~s}, 2 \mathrm{H}), 3.56(\mathrm{~s}, 2 \mathrm{H}), 2.83(\mathrm{t}, J=5.8 \mathrm{~Hz}, 2 \mathrm{H})$, $2.69(\mathrm{t}, J=5.8 \mathrm{~Hz}, 2 \mathrm{H}) .{ }^{13} \mathrm{C}-\mathrm{NMR}\left(150 \mathrm{MHz}, \mathrm{DMSO}-d_{6}\right) \delta 165.6,142.6,138.7,134.7,134.1,133.5,131.5$, 128.7, 128.5, 127.8, 126.4, 126.1, 125.6, 122.2, 115.3, 61.4, 55.5, 50.3, 39.5, 28.7. ESI-MS $m / z 421.0[\mathrm{M}+\mathrm{H}]^{+}$. HR-ESIMS: $[\mathrm{M}+\mathrm{H}]^{+}$calcd for $\mathrm{C}_{23} \mathrm{H}_{22} \mathrm{BrN}_{2} \mathrm{O}^{+} 421.0916$, found 421.0922 . 
2-bromophenyl 4-((3,4-dihydroisoquinolin-2(1H)-yl)methyl)benzoate (26). Yellow oil (yield 64\%). ${ }^{1} \mathrm{H}-\mathrm{NMR}$ $\left(600 \mathrm{MHz}, \mathrm{DMSO}-d_{6}\right) \delta 8.14(\mathrm{~d}, J=8.3 \mathrm{~Hz}, 2 \mathrm{H}), 7.78(\mathrm{dd}, J=8.1,1.5 \mathrm{~Hz}, 1 \mathrm{H}), 7.63(\mathrm{~d}, J=8.3 \mathrm{~Hz}, 2 \mathrm{H})$, 7.52-7.49 (m, 1H), $7.46(\mathrm{dd}, J=8.1,1.5 \mathrm{~Hz}, 1 \mathrm{H}), 7.31-7.28(\mathrm{~m}, 1 \mathrm{H}), 7.13-7.08(\mathrm{~m}, 3 \mathrm{H}), 7.02(\mathrm{~d}, J=7.3$ $\mathrm{Hz}, 1 \mathrm{H}), 3.78(\mathrm{~s}, 2 \mathrm{H}), 3.58(\mathrm{~s}, 2 \mathrm{H}), 2.84(\mathrm{t}, J=5.8 \mathrm{~Hz}, 2 \mathrm{H}), 2.72(\mathrm{t}, J=5.8 \mathrm{~Hz}, 2 \mathrm{H}) .{ }^{13} \mathrm{C}-\mathrm{NMR}(150 \mathrm{MHz}$, DMSO- $\left.d_{6}\right) \delta 163.6,148.0,145.7,134.6,134.0,133.2,130.1,129.2,128.5,128.1,127.0,126.4,126.0,125.5$, 124.5, 115.7, 61.3, 55.4, 50.3, 39.5, 28.7. ESI-MS $m / z 422.0[\mathrm{M}+\mathrm{H}]^{+}$. HR-ESIMS: $[\mathrm{M}+\mathrm{H}]^{+}$calcd for $\mathrm{C}_{23} \mathrm{H}_{21} \mathrm{BrNO}_{2}{ }^{+}$422.0756, found 422.0757 .

S-(2-bromophenyl) 4-((3,4-dihydroisoquinolin-2(1H)-yl)methyl)benzothioate (27). Yellow oil (yield 61.3\%). ${ }^{1} \mathrm{H}-\mathrm{NMR}\left(600 \mathrm{MHz}\right.$, DMSO- $\left.d_{6}\right) \delta 7.99(\mathrm{~d}, J=8.3 \mathrm{~Hz}, 2 \mathrm{H}), 7.86(\mathrm{dd}, J=7.9,1.3 \mathrm{~Hz}, 1 \mathrm{H}), 7.71(\mathrm{dd}, J=7.6$, $1.7 \mathrm{~Hz}, 1 \mathrm{H}), 7.61(\mathrm{~d}, J=8.3 \mathrm{~Hz}, 2 \mathrm{H}), 7.54-7.51(\mathrm{~m}, 1 \mathrm{H}), 7.49-7.46(\mathrm{~m}, 1 \mathrm{H}), 7.13-7.07(\mathrm{~m}, 3 \mathrm{H}), 7.01(\mathrm{~d}, J=7.3$ $\mathrm{Hz}, 1 \mathrm{H}), 3.76(\mathrm{~s}, 2 \mathrm{H}), 3.57(\mathrm{~s}, 2 \mathrm{H}), 2.83(\mathrm{t}, J=5.8 \mathrm{~Hz}, 2 \mathrm{H}), 2.70(\mathrm{t}, J=5.8 \mathrm{~Hz}, 2 \mathrm{H}) .{ }^{13} \mathrm{C}-\mathrm{NMR}(150 \mathrm{MHz}$, DMSO- $\left.d_{6}\right) \delta 187.1,145.9,138.0,134.6,134.3,134.0,133.5,132.1,129.6,129.4,128.7,128.5,128.3,127.4$, 126.4, 126.0, 125.5, 61.2, 55.4, 50.3, 28.7. ESI-MS $m / z 438.0[\mathrm{M}+\mathrm{H}]^{+}$. HR-ESIMS: $[\mathrm{M}+\mathrm{H}]^{+}$calcd for $\mathrm{C}_{23} \mathrm{H}_{21}$ BrNOS $^{+}$438.0527, found 438.0557 .

$\mathrm{N}$-(2-(1H-indol-3-yl)ethyl)-4-((3,4-dihydroisoquinolin-2(1H)-yl)methyl)benzamide (28). ${ }^{1} \mathrm{H}-\mathrm{NMR}(600 \mathrm{MHz}$, DMSO- $\left.d_{6}\right) \delta 10.81(\mathrm{brs}, 1 \mathrm{H}, \mathrm{NH}), 8.58(\mathrm{t}, J=5.7 \mathrm{~Hz}, 1 \mathrm{H}), 7.83(\mathrm{~d}, J=8.2 \mathrm{~Hz}, 2 \mathrm{H}), 7.59(\mathrm{~d}, J=8.0 \mathrm{~Hz}, 1 \mathrm{H})$, $7.44(\mathrm{~d}, J=8.2 \mathrm{~Hz}, 2 \mathrm{H}), 7.34(\mathrm{~d}, J=8.0 \mathrm{~Hz}, 1 \mathrm{H}), 7.18(\mathrm{~d}, J=2.2 \mathrm{~Hz}, 1 \mathrm{H}), 7.15-7.03(\mathrm{~m}, 4 \mathrm{H}), 7.02-6.93$ $(\mathrm{m}, 2 \mathrm{H}), 3.69(\mathrm{~s}, 2 \mathrm{H}), 3.58-3.51(\mathrm{~m}, 4 \mathrm{H}), 2.95(\mathrm{t}, J=7.5 \mathrm{~Hz}, 2 \mathrm{H}), 2.82(\mathrm{t}, J=5.8 \mathrm{~Hz}, 2 \mathrm{H}), 2.68(\mathrm{t}, J=5.8 \mathrm{~Hz}$, 2H). ${ }^{13}$ C-NMR $\left(150 \mathrm{MHz}\right.$, DMSO-d $\left.d_{6}\right) \delta 166.0,141.7,136.3,134.7,134.1,133.5,128.5,127.3,127.2,126.4$, $126.0,125.5,122.6,120.9,118.3,118.2,111.9,111.4,61.4,55.5,50.3,40.2,39.5,28.7,25.2$. ESI-MS $m / z 410.1$ $[\mathrm{M}+\mathrm{H}]^{+}$. HR-ESIMS: $[\mathrm{M}+\mathrm{H}]^{+}$calcd for $\mathrm{C}_{27} \mathrm{H}_{28} \mathrm{~N}_{3} \mathrm{O}^{+}$410.2232, found 410.2250.

4-((3,4-dihydroisoquinolin-2(1H)-yl)methyl)-N-(2-(5-methyl-1H-indol-3-yl)ethyl)benzamide (29). White solid (yield 63.2\%). ${ }^{1} \mathrm{H}-\mathrm{NMR}\left(600 \mathrm{MHz}\right.$, DMSO- $\left.d_{6}\right) \delta 10.67$ (brs, $\left.1 \mathrm{H}, \mathrm{NH}\right), 8.57(\mathrm{t}, J=5.6 \mathrm{~Hz}, 1 \mathrm{H}), 7.83$ $(\mathrm{d}, J=8.2 \mathrm{~Hz}, 2 \mathrm{H}), 7.44(\mathrm{~d}, J=8.2 \mathrm{~Hz}, 2 \mathrm{H}), 7.34(\mathrm{~s}, 1 \mathrm{H}), 7.22(\mathrm{~d}, J=8.2 \mathrm{~Hz}, 1 \mathrm{H}), 7.16-7.04(\mathrm{~m}, 4 \mathrm{H}), 6.99$ $(\mathrm{d}, J=7.2 \mathrm{~Hz}, 1 \mathrm{H}), 6.89(\mathrm{dd}, J=8.2,1.2 \mathrm{~Hz}, 1 \mathrm{H}), 3.69(\mathrm{~s}, 2 \mathrm{H}), 3.53(\mathrm{~d}, J=7.8 \mathrm{~Hz}, 4 \mathrm{H}), 2.92(\mathrm{t}, J=7.5 \mathrm{~Hz}$, 2H), $2.82(\mathrm{t}, J=5.8 \mathrm{~Hz}, 2 \mathrm{H}), 2.68(\mathrm{t}, J=5.8 \mathrm{~Hz}, 2 \mathrm{H}), 2.35(\mathrm{~s}, 3 \mathrm{H}) .{ }^{13} \mathrm{C}-\mathrm{NMR}\left(150 \mathrm{MHz}, \mathrm{DMSO}-d_{6}\right) \delta 166.0$, 141.7, 134.7, 134.6, 134.1, 133.5, 128.5, 128.4, 127.6, 127.2, 126.5, 126.3, 126.0, 125.5, 122.7, 122.5, 118.0, 111.5, 111.1, 61.4, 55.4, 50.3, 40.4, 39.5, 28.7, 25.2, 21.3. ESI-MS m/z 424.2 [M + H] ${ }^{+}$. HR-ESIMS: [M + H] ${ }^{+}$ calcd for $\mathrm{C}_{28} \mathrm{H}_{30} \mathrm{~N}_{3} \mathrm{O}^{+} 424.2389$, found 424.2404 .

4-((3,4-dihydroisoquinolin-2(1H)-yl)methyl)-N-(2-(5-methoxy-1H-indol-3-yl)ethyl)benzamide (30). White solid (yield 58\%). ${ }^{1} \mathrm{H}-\mathrm{NMR}\left(600 \mathrm{MHz}, \mathrm{DMSO}-d_{6}\right) \delta 10.64$ (brs, $\left.1 \mathrm{H}, \mathrm{NH}\right), 8.57(\mathrm{t}, J=5.7 \mathrm{~Hz}, 1 \mathrm{H}), 7.83$ $(\mathrm{d}, J=8.3 \mathrm{~Hz}, 2 \mathrm{H}), 7.44(\mathrm{~d}, J=8.3 \mathrm{~Hz}, 2 \mathrm{H}), 7.22(\mathrm{~d}, J=8.8 \mathrm{~Hz}, 1 \mathrm{H}), 7.14(\mathrm{~d}, J=2.3 \mathrm{~Hz}, 1 \mathrm{H}), 7.13-7.06(\mathrm{~m}$, $3 \mathrm{H}), 7.05(\mathrm{~d}, J=2.3 \mathrm{~Hz}, 1 \mathrm{H}), 6.99(\mathrm{~d}, J=7.2 \mathrm{~Hz}, 1 \mathrm{H}), 6.71(\mathrm{dd}, J=8.7,2.4 \mathrm{~Hz}, 1 \mathrm{H}), 3.71(\mathrm{~s}, 3 \mathrm{H}), 3.69$ $(\mathrm{s}, 2 \mathrm{H}), 3.57-3.50(\mathrm{~m}, 4 \mathrm{H}), 2.92(\mathrm{t}, J=7.4 \mathrm{~Hz}, 2 \mathrm{H}), 2.82(\mathrm{t}, J=5.8 \mathrm{~Hz}, 2 \mathrm{H}), 2.70-2.65(\mathrm{~m}, 2 \mathrm{H}) .{ }^{13} \mathrm{C}-\mathrm{NMR}$ $\left(150 \mathrm{MHz}, \mathrm{DMSO}-d_{6}\right) \delta 166.0,153.0,141.7,134.7,134.1,133.5,131.4,128.5,128.5,127.7,127.2,126.4$, 126.0, 125.5, 123.3, 112.0, 111.8, 111.1, 100.2, 61.4, 55.4, 55.3, 50.3, 48.6, 40.2, 39.5, 28.7, 25.2. ESI-MS m/z $440.2[\mathrm{M}+\mathrm{H}]^{+}$. HR-ESIMS: $[\mathrm{M}+\mathrm{H}]^{+}$calcd for $\mathrm{C}_{28} \mathrm{H}_{30} \mathrm{~N}_{3} \mathrm{O}_{2}{ }^{+} 440.2338$, found 440.2324.

\subsection{BChE and AChE Bioassay}

BChE (EC 3.1.1.8, from human serum), AChE (EC 3.1.1.8, from human erythrocyte), butyrylthiocholine (BUC) iodide, and acetylthiocholine (ATC) iodide were purchased from Sigma-Aldrich (St. Louis, MO, USA). Enzyme was dissolved and pre-prepared at 2.0 U/mL. $10 \mu \mathrm{L}$ of enzyme, $20 \mu \mathrm{L}$ of $0.01 \mathrm{M}$ 5,5'-dithiobis(2-nitrobenzoic acid), $10 \mu \mathrm{L}$ of tested compound, and $40 \mu \mathrm{L}$ of PBS were pre-incubated together for $5 \mathrm{~min}$. Io initiate the reaction, $20 \mu \mathrm{L}$ of $0.01 \mathrm{M} \mathrm{BUC}$ or ATC was added. The activity was determined by measuring the increase in absorbance at $410 \mathrm{~nm}$ at $37^{\circ} \mathrm{C}$ in $2 \mathrm{~min}$ intervals using Tecan Spark multimode microplate reader (Mannedorf, Switzerland). The percentage of inhibition (I) was calculated according to the formula: $\mathrm{I}=(\mathrm{Ac}-\mathrm{Ai}) / \mathrm{Ac} \times 100 \%$, with Ai and Ac 
representing the change in the absorbance in the presence of an inhibitor and without an inhibitor, respectively $[28,29]$.

\subsection{Kinetic Assay}

Kinetic study of BChE inhibition was performed by using Ellman's method [28,29] as described in Section 3.3.

\subsection{Molecular Docking}

The molecular docking procedure to study the binding mode of 23 against BChE was performed according to our previously reported protocol [28,29].

\subsection{Molecular Dynamics Simulation}

After the BChE:23 complex was obtained by molecular docking, MD simulation was subsequently employed to probe this complex. The protonation states of ionizable residues in $\mathrm{BChE}$ were determined using the $\mathrm{H}++$ program [48]. A periodic box of TIP3P [49] water molecules that extend $10 \AA$ from the protein atoms was added. Then counter-ions were added to neutralize the simulation model. The AMBER force field (parm99SB) was used on the protein. The restrained electrostatic potential method [50] encoded in the AMBER suite of programs [51] at the RHF/6-31G* level was used to calculate the atom charges of 23 . Those already present in the AMBER force field covalent and nonbonded parameters were assigned for 23.

All the MD simulations (at constant temperature and pressure and periodic boundary conditions) were conducted with SANDER program (time step $=2 \mathrm{fs}$ ). All hydrogen atom bonds in the simulation system were constrained by the SHAKE algorithm [52]. The particle-mesh Ewald method was used to calculate electrostatic interactions [53].

\subsection{Binding Free Energy Calculation with MM-PBSA Method}

The binding free energy of $\mathbf{2 3}$ with BChE was calculated using the MM-PBSA method encoded in the AMBER 10.0 program. Based on the 100 ns equilibrated dynamic trajectory, 1000 snapshots from the trajectory were extracted every $100 \mathrm{ps}$, and were used for the binding free energy calculation. The MMPBSA.PY module in AMBER 10.0 was employed to perform the calculation.

\subsection{Determination of the Inhibitory Potency on $A \beta_{1-42}$ Aggregation}

The ThT-based fluorometric assay [54] was performed for the determination of compounds' anti-A $\beta_{1-42}$ aggregation potency. The detailed procedure can be found in Supplementary Materials.

\subsection{Cell Viability Assay and Neuroprotective Activity Evaluation}

The MTT method [55] was used to test the cytotoxicity and neuroprotective activity evaluation of compounds. The detailed procedure was included in Supplementary Materials.

\subsection{Statistical Analyses}

All of the experimental values were expressed as mean \pm standard deviation (SD) of at least three independent experiments. GraphPad Prism 5.0 statistical software package (GraphPad Software, San Diego, CA, USA) was used to perform all of the statistical analysis.

\section{Conclusions}

In the present study, a series of novel selective BChE inhibitors were derived from the structural optimization of hit $\mathbf{1}$. Compounds $\mathbf{9}$ and $\mathbf{2 3}$ displayed improved BChE inhibitory activity and good selectivity towards BChE. The current preliminary SAR study mainly concerned the effects of amide side-chain and substituents at tetrahydroisoquinoline on the BChE inhibitory activity. From the bioassay 
results, it could be summarized as follows: (1) for compounds 1-10, tetrahydropyrrole ring could improve the BChE activity, (2) for compounds 11-14, the substitution at C-6 and C-7 position of tetrahydroisoquinoline led to the decrease of BChE activity and selectivity, and (3) for compounds 15-30, the substitution at C-2 of benzene ring should be optimal for BChE activity and amide group was found to be greatly important for the binding of inhibitor to BChE. Kinetic analysis together with molecular modeling studies suggested that these derivatives are a mixed-type of BChE inhibitors. Further, the selected compounds 9 and 23 displayed inhibitory activity against $A \beta_{1-42}$ aggregation by a dose-dependent manner. Moreover, these two compounds are non-toxic towards SH-SY5Y cells even at $200 \mu \mathrm{M}$, indicating they are probably safe on SH-SY5Y cells. Of course, this discussion on safety needs more in vitro and even in vivo evaluations. More importantly, compounds 9 and 23 showed significantly in vitro protective activity against $A \beta_{1-42}$-induced toxicity in SH-SY5Y cell model at 5 and $10 \mu \mathrm{M}$, and compound 23 even showed much better activity than the positive control EGCG at $10 \mu \mathrm{M}$. In summary, the present in vitro biological evaluation together with computational analyses provided a new valuable chemical template, which deserves further optimization for an anti-AD drug discovery.

Supplementary Materials: The following are available online, Figure S1, ${ }^{1} \mathrm{H},{ }^{13} \mathrm{C}$, HR-ESIMS spectra for compounds 1-30, methods for determination of the inhibitory potency on $\mathrm{A} \beta_{1-42}$ aggregation, Cell viability assay, neuroprotective activity evaluation.

Author Contributions: Chemical synthesis, ChE inhibition and anti-A $\beta$ aggregation test, C.-S.J. and Y.-X.G.; Cell viability assay, Z.-Q.C.; spectroscopic measurements, Y.-Y.W.; molecular docking and analysis, K.Z. and H.-R.T.; Writing-Original Draft Preparation, C.-S.J. and K.Z.; Writing-Review, H.Z.; Project Administration, C.-S.J., and K.Z. All the authors accepted the final version of the manuscript.

Funding: This research was funded by [National Natural Science Foundation of China] grant numbers [21672082, 81803438]; [Natural Science Foundation of Shandong Province] grant numbers [ZR2019YQ31, ZR2017BH038, JQ201721, ZR2018ZC0944]; [Young Taishan Scholars Program] grant number [tsqn20161037]; and [Shandong Talents Team Cultivation Plan of University Preponderant Discipline] grant number [10027].

Conflicts of Interest: The authors declare no conflict of interest.

\section{Abbreviations}

$\begin{array}{ll}\text { Alzheimer's disease } & (\mathrm{AD}) \\ \text { acetylcholine } & (\mathrm{ACh}) \\ \text { beta-amyloid } & (\mathrm{A} \beta) \\ \text { cholinesterase } & (\mathrm{ChE}) \\ \text { butyrylcholinesterase } & (\mathrm{BChE}) \\ \text { acetylcholinesterase } & (\mathrm{AChE}) \\ \text { catalytic active site } & (\mathrm{CAS}) \\ \text { peripheral anionic site } & (\mathrm{PAS}) \\ \text { structure-activity relationship } & (\mathrm{SAR}) \\ \text { molecular dynamics } & (\mathrm{MD}) \\ \text { Molecular Mechanics Poisson-Boltzmann Surface Area } \\ \text { root-mean-square deviations } & (\mathrm{MM}-\mathrm{PBSA}) \\ \text { thioflavin T } & (\mathrm{RMSDs}) \\ \text { epigallocatechin gallate } & \text { (ThT) } \\ \text { butyrylthiocholine } & \text { (EGCG) } \\ \text { acetylthiocholine } & \text { (BUC) }\end{array}$

\section{References}

1. Wiemann, J.; Loesche, A.; Csuk, R. Novel dehydroabietylamine derivatives as potent inhibitors of acetylcholinesterase. Bioorg. Chem. 2017, 74, 145-157. [CrossRef] [PubMed]

2. Mattila, J.; Soininen, H.; Koikkalainen, J.; Rueckert, D.; Wolz, R.; Waldemar, G.; Lötjönen, J. Optimizing the diagnosis of early Alzheimer's disease in mild cognitive impairment subjects. J. Alzheimer's Dis. 2012, 32, 969-979. [CrossRef] [PubMed] 
3. Available online: https://www.alz.co.uk/research/world-report-2018 (accessed on 20 May 2019).

4. Yu, Y.F.; Huang, Y.D.; Zhang, C.; Wu, X.N.; Zhou, Q.; Wu, D.; Wu, Y.; Luo, H.B. Discovery of Novel Pyrazolopyrimidinone Derivatives as Phosphodiesterase 9A Inhibitors Capable of Inhibiting Butyrylcholinesterase for Treatment of Alzheimer's Disease. ACS Chem. Neurosci. 2017, 8, 2522-2534. [CrossRef] [PubMed]

5. Lu, X.; Yang, H.; Li, Q.; Chen, Y.; Li, Q.; Zhou, Y.; Feng, F.; Liu, W.; Guo, Q.; Sun, H. Expansion of the scaffold diversity for the development of highly selective butyrylcholinesterase (BChE) inhibitors: Discovery of new hits through the pharmacophore model generation, virtual screening and molecular dynamics simulation. Bioorg. Chem. 2019, 85, 117-127. [CrossRef] [PubMed]

6. Scarpini, E.; Scheltens, P.; Feldman, H. Treatment of Alzheimer's disease: Current status and new perspectives. Lancet Neurol. 2003, 2, 539-547. [CrossRef]

7. Congdon, E.E.; Sigurdsson, E.M. Tau-targeting therapies for Alzheimer disease. Nat. Rev. Neurol. 2018, 14, 399-415. [CrossRef]

8. Kodamullil, A.T.; Zekri, F.; Sood, M.; Hengerer, B.; Canard, L.; McHale, D.; Hofmann-Apitius, M. Trial watch: Tracing investment in drug development for Alzheimer disease. Nat. Rev. Drug Discov. 2017, 16, 819. [CrossRef]

9. Bartus, R.T.; Dean, R.L.; Beer, B.; Lippa, A.S. The cholinergic hypothesis of geriatric memory dysfunction. Science 1982, 217, 408-414. [CrossRef]

10. Anand, P.; Singh, B. A review on cholinesterase inhibitors for Alzheimer's disease. Arch Pharm. Res. 2013, 36, 375-399. [CrossRef]

11. Feng, B.; Li, X.; Xia, J.; Wu, S. Discovery of novel isoflavone derivatives as AChE/BuChE dual-targeted inhibitors: Synthesis, biological evaluation and molecular modelling. J. Enzyme Inhib. Med. Chem. 2017, 32, 968-977. [CrossRef]

12. De Vita, D.; Pandolfi, F.; Ornano, L.; Feroci, M.; Chiarotto, I.; Sileno, I.; Pepi, F.; Costi, R.; Di Santo, R.; Scipione, L. New N,N-dimethylcarbamate inhibitors of acetylcholinesterase: Design synthesis and biological evaluation. J. Enzyme Inhib. Med. Chem. 2016, 31, 106-113. [CrossRef] [PubMed]

13. Colović, M.B.; Krstić, D.Z.; Lazarević-Pašti, T.D.; Bondžić, A.M.; Vasić, V.M. Acetylcholinesterase inhibitors: pharmacology and toxicology. Curr. Neuropharmacol. 2013, 11, 315-335. [CrossRef] [PubMed]

14. Perry, E.K.; Tomlinson, B.E.; Blessed, G.; Bergmann, K.; Gibson, P.H.; Perry, R.H. Correlation of cholinergic abnormalities with senile plaques and mental test scores in senile dementia. Br. Med. J. 1978, 2, 1457-1459. [CrossRef] [PubMed]

15. Greig, N.H.; Utsuki, T.; Ingram, D.K.; Wang, Y.; Pepeu, G.; Scali, C.; Yu, Q.S.; Mamczarz, J.; Holloway, H.W.; Giordano, T.; et al. Selective butyrylcholinesterase inhibition elevates brain acetylcholine, augments learning and lowers Alzheimer beta-amyloid peptide in rodent. Proc. Natl. Acad. Sci. USA 2005, 102, 17213-17218. [CrossRef] [PubMed]

16. Li, Q.; Yang, H.; Chen, Y.; Sun, H. Recent progress in the identification of selective butyrylcholinesterase inhibitors for Alzheimer's disease. Eur. J. Med. Chem. 2017, 32, 294-309. [CrossRef] [PubMed]

17. Petersen, R.C.; Thomas, R.G.; Grundman, M.; Bennett, D.; Doody, R.; Ferris, S.; Galasko, D.; Jin, S.; Kaye, J.; Levey, A.; et al. Vitamin E and donepezil for the treatment of mild cognitive impairment. N. Engl. J. Med. 2005, 352, 2379-2388. [CrossRef]

18. Dileep, K.V.; Remya, C.; Tintu, I.; Sadasivan, C. Inhibition, ADME and structure based modification of IAA and IBA against acetylcholinesterase: An attempt towards new drug development for Alzheimer's disease. Front. Life Sci. 2013, 7, 164-173. [CrossRef]

19. Li, B.; Duysen, E.G.; Carlson, M.; Lockridge, O. The butyrylcholinesterase knockout mouse as a model for human butyrylcholinesterase deficiency. J. Pharmacol. Exp. Ther. 2008, 324, 1146-1154. [CrossRef]

20. Hartmann, J.; Kiewert, C.; Duysen, E.G.; Lockridge, O.; Greig, N.H.; Klein, J. Excessive hippocampal acetylcholine levels in acetylcholinesterase-deficient mice are moderated by butyrylcholinesterase activity. J. Neurochem. 2007, 100, 1421-1429. [CrossRef]

21. Fang, L.; Pan, Y.; Muzyka, J.L.; Zhan, C.G. Active site gating and substrate specificity of butyrylcholinesterase and acetylcholinesterase: Insights from molecular dynamics simulations. J. Phys. Chem. B 2011, 115, 8797-8805. [CrossRef] 
22. Sawatzky, E.; Wehle, S.; Kling, B.; Wendrich, J.; Bringmann, G.; Sotriffer, C.A.; Heilmann, J.; Decker, M. Discovery of Highly Selective and Nanomolar Carbamate-Based Butyrylcholinesterase Inhibitors by Rational Investigation into Their Inhibition Mode. J. Med. Chem. 2016, 59, 2067-2082. [CrossRef] [PubMed]

23. Heller, L.; Kahnt, M.; Loesche, A.; Grabandt, P.; Schwarz, S.; Brandt, W.; Csuk, R. Amino derivatives of platanic acid act as selective and potent inhibitors of butyrylcholinesterase. Eur. J. Med. Chem. 2017, 126, 652-668. [CrossRef] [PubMed]

24. Košak, U.; Brus, B.; Knez, D.; Žakelj, S.; Trontelj, J.; Pišlar, A.; Šink, R.; Jukič, M.; Živin, M.; Podkowa, A.; et al. The Magic of Crystal Structure-Based Inhibitor Optimization: Development of a Butyrylcholinesterase Inhibitor with Picomolar Affinity and in Vivo Activity. J. Med. Chem. 2018, 61, 119-139. [CrossRef] [PubMed]

25. Yu, Q.; Holloway, H.W.; Utsuki, T.; Brossi, A.; Greig, N.H. Synthesis of Novel Phenserine-Based-Selective Inhibitors of Butyrylcholinesterase for Alzheimer's Disease. J. Med. Chem. 1999, 42, 1855-1861. [CrossRef] [PubMed]

26. Carolan, C.G.; Dillon, G.P.; Khan, D.; Ryder, S.A.; Gaynor, J.M.; Reidy, S.; Marquez, J.F.; Jones, M.; Holland, V.; Gilmer, J.F. Isosorbide-2-benzyl carbamate-5-salicylate, a peripheral anionic site binding subnanomolar selective butyrylcholinesterase inhibitor. J. Med. Chem. 2010, 53, 1190-1199. [CrossRef] [PubMed]

27. Jing, L.; Wu, G.; Kang, D.; Zhou, Z.; Song, Y.; Liu, X.; Zhan, P. Contemporary medicinal-chemistry strategies for the discovery of selective butyrylcholinesterase inhibitors. Drug Discov. Today 2019, 24, 629-635. [CrossRef] [PubMed]

28. Neves, B.J.; Braga, R.C.; Melo-Filho, C.C.; Moreira-Filho, J.T.; Muratov, E.N.; Andrade, C.H. QSAR-Based Virtual Screening: Advances and Applications in Drug Discovery. Front. Pharmacol. 2018, 9, 1275. [CrossRef]

29. Beesu, M.; Caruso, G.; Salyer, A.C.; Khetani, K.K.; Sil, D.; Weerasinghe, M.; Tanji, H.; Ohto, U.; Shimizu, T.; David, S.A. Structure-Based Design of Human TLR8-Specific Agonists with Augmented Potency and Adjuvanticity. J. Med. Chem. 2015, 58, 7833-7849. [CrossRef] [PubMed]

30. Beesu, M.; Caruso, G.; Salyer, A.C.; Shukla, N.M.; Khetani, K.K.; Smith, L.J.; Fox, L.M.; Tanji, H.; Ohto, U.; Shimizu, T.; et al. Identification of a Human Toll-like Receptor (TLR) 8-Specific Agonist and a Functional Pan-TLR inhibitor in 2-Aminoimidazoles. J. Med. Chem. 2016, 59, 3311-3330. [CrossRef]

31. Cheng, Z.Q.; Zhu, K.K.; Zhang, J.; Song, J.L.; Muehlmann, L.A.; Jiang, C.S.; Liu, C.L.; Zhang, H. Molecular-docking-guided design and synthesis of new IAA-tacrine hybrids as multifunctional $\mathrm{AChE} / \mathrm{BChE}$ inhibitors. Bioorg. Chem. 2019, 83, 277-288. [CrossRef]

32. Cheng, Z.Q.; Song, J.L.; Zhu, K.; Zhang, J.; Jiang, C.S.; Zhang, H. Total Synthesis of Pulmonarin B and Design of Brominated Phenylacetic Acid/Tacrine Hybrids: Marine Pharmacophore Inspired Discovery of New ChE and A $\beta$ Aggregation Inhibitors. Mar. Drugs 2018, 16, 293. [CrossRef] [PubMed]

33. Zhang, J.; Li, J.C.; Song, J.L.; Cheng, Z.Q.; Sun, J.Z.; Jiang, C.S. Synthesis and evaluation of coumarin/1,2,4-oxadiazole hybrids as selective BChE inhibitors with neuroprotective activity. J. Asian Nat. Prod. 2018. [CrossRef] [PubMed]

34. Zhang, J.; Jiang, C.S. Synthesis and evaluation of coumarin/piperazine hybrids as acetylcholinesterase inhibitors. Med. Chem. Res. 2018, 27, 1717-1727. [CrossRef]

35. Li, J.C.; Zhang, J.; Rodrigues, M.C.; Ding, D.J.; Longo, J.P.; Azevedo, R.B.; Muehlmann, L.A.; Jiang, C.S. Synthesis and evaluation of novel 1,2,3-triazole-based acetylcholinesterase inhibitors with neuroprotective activity. Bioorg. Med. Chem. Lett. 2016, 26, 3881-3885. [CrossRef] [PubMed]

36. Jiang, C.S.; Ge, Y.X.; Cheng, Z.Q.; Song, J.L.; Wang, Y.Y.; Zhu, K.; Zhang, H. Discovery of new multifunctional selective acetylcholinesterase inhibitors: Structure-based virtual screening and biological evaluation. J. Comput. Aided Mol. Des. 2019, 33, 521-530. [CrossRef] [PubMed]

37. Nachon, F.; Carletti, E.; Ronco, C.; Trovaslet, M.; Nicolet, Y.; Jean, L.; Renard, P.Y. Crystal structures of human cholinesterases in complex with huprine $\mathrm{W}$ and tacrine: Elements of specificity for anti-Alzheimer's drugs targeting acetyl- and butyryl-cholinesterase. Biochem. J. 2013, 453, 393-399. [CrossRef] [PubMed]

38. Ghafary, S.; Najafi, Z.; Mohammadi-Khanaposhtani, M.; Nadri, H.; Edraki, N.; Ayashi, N.; Larijani, B.; Amini, M.; Mahdavi, M. Novel cinnamic acid-tryptamine hybrids as potent butyrylcholinesterase inhibitors: Synthesis, biological evaluation, and docking study. Arch. Pharm. 2018, 351, e1800115. [CrossRef]

39. Razzokov, J.; Yusupov, M.; Bogaerts, A. Oxidation destabilizes toxic amyloid beta peptide aggregation. Sci. Rep. 2019, 9, 5476. [CrossRef]

40. Yuan, C.; Gao, Z. A $\beta$ interacts with both the iron center and the porphyrin ring of heme: Mechanism of heme's action on A $\beta$ aggregation and disaggregation. Chem. Res. Toxicol. 2013, 26, 262-269. [CrossRef] 
41. Caruso, G.; Distefano, D.A.; Parlascino, P.; Fresta, C.G.; Lazzarino, G.; Lunte, S.M.; Nicoletti, V.G. Receptor-mediated toxicity of human amylin fragment aggregated by short- and long-term incubations with copper ions. Mol. Cell. Biochem. 2017, 425, 85-93. [CrossRef]

42. Selkoe, D.J. Soluble oligomers of the amyloid beta-protein impair synaptic plasticity and behavior. Behav. Brain Res. 2008, 192, 106-113. [CrossRef] [PubMed]

43. Ramanan, V.K.; Risacher, S.L.; Nho, K.; Kim, S.; Swaminathan, S.; Shen, L.; Foroud, T.M.; Hakonarson, H.; Huentelman, M.J.; Aisen, P.S.; et al. APOE and BCHE as modulators of cerebral amyloid deposition: A florbetapir PET genome-wide association study. Mol. Psychiatry 2014, 19, 351-357. [CrossRef] [PubMed]

44. Reid, G.A.; Darvesh, S. Butyrylcholinesterase-knockout reduces brain deposition of fibrillar $\beta$-amyloid in an Alzheimer mouse model. Neuroscience 2015, 298, 424-435. [CrossRef] [PubMed]

45. Friesner, R.A.; Banks, J.L.; Murphy, R.B.; Halgren, T.A.; Klicic, J.J.; Mainz, D.T.; Repasky, M.P.; Knoll, E.H.; Shelley, M.; Perry, J.K.; et al. Glide: A new approach for rapid, accurate docking and scoring. 1. Method and assessment of docking accuracy. J. Med. Chem. 2004, 47, 1739-1749. [CrossRef] [PubMed]

46. Halgren, T.A.; Murphy, R.B.; Friesner, R.A.; Beard, H.S.; Frye, L.L.; Pollard, W.T.; Banks, J.L. Glide: A new approach for rapid, accurate docking and scoring. 2. Enrichment factors in database screening. J. Med. Chem. 2004, 47, 1750-1759. [CrossRef]

47. Friesner, R.A.; Murphy, R.B.; Repasky, M.P.; Frye, L.L.; Greenwood, J.R.; Halgren, T.A.; Sanschagrin, P.C.; Mainz, D.T. Extra precision glide: Docking and scoring incorporating a model of hydrophobic enclosure for protein-ligand complexes. J. Med. Chem. 2006, 49, 6177-6196. [CrossRef] [PubMed]

48. Gordon, J.C.; Myers, J.B.; Folta, T.; Shoja, V.; Heath, L.S.; Onufriev, A. H++: A server for estimating pKas and adding missing hydrogens to macromolecules. Nucleic Acids Res. 2005, 33, W368-W371. [CrossRef]

49. Jorgensen, W.L.; Chandrasekhar, J.; Madura, J.D.; Impey, R.W.; Klein, M.L. Comparison of simple potential functions for simulating liquid water. J. Chem. Phys. 1983, 79, 926-935. [CrossRef]

50. Bayly, C.I.; Cieplak, P.; Cornell, W.D.; Kollman, P.A. A well-behaved electrostatic potential based method using charge restraints for deriving atomic charges: The RESP model. J. Phys. Chem. 1993, 97, 10269-10280. [CrossRef]

51. Case, D.A.; Cheatham, T.E.; Darden, T.; Gohlke, H.; Luo, R.; Merz, K.M.; Onufriev, A.; Simmerling, C.; Wang, B.; Woods, R.J. The Amber biomolecular simulation programs. J. Comput. Chem. 2005, 26, 1668-1688. [CrossRef]

52. Ryckaert, J.P.; Ciccotti, G.; Berendsen, H.J.C. Numerical integration of the cartesian equations of motion of a system with constraints: molecular dynamics of n-alkanes. J. Comput. Phys. 1977, 23, 327-341. [CrossRef]

53. Darden, T.; York, D.; Pedersen, L. Numerical-integration of Cartesian equations of motion of a system with constraints: Molecular-dynamics of nalkanes. J. Chem. Phys. 1993, 98, 10089-10092. [CrossRef]

54. Fang, L.; Fang, X.; Gou, S.; Lupp, A.; Lenhardt, I.; Sun, Y.; Huang, Z.; Chen, Y.; Zhang, Y.; Fleck, C. Design, synthesis and biological evaluation of D-ring opened galantamine analogs as multifunctional anti-Alzheimer agents. Eur. J. Med. Chem. 2014, 76, 376-386. [CrossRef] [PubMed]

55. Mei, W.W.; Ji, S.S.; Xiao, W.; Wang, X.D.; Jiang, C.S.; Ma, W.Q.; Zhang, H.Y.; Gong, J.X.; Guo, Y.W. Synthesis and biological evaluation of benzothiazol-based 1,3,4-oxadiazole derivatives as amyloid $\beta$-targeted compounds against Alzheimer's disease. Monatsh. Chem. 2017, 148, 1807-1815. [CrossRef]

Sample Availability: Samples of the compounds 1-30 are available from the authors. 